\title{
The evolution of planetary nebulae
}

\section{A radiation-hydrodynamics parameter study}

\author{
M. Perinotto ${ }^{1}$, D. Schönberner ${ }^{2}$, M. Steffen ${ }^{2}$, and C. Calonaci $^{3}$ \\ 1 Dipartimento di Astronomia e Scienza dello Spazio, Largo E. Fermi 2, 50125 Firenze, Italy \\ e-mail: mariop@arcetri.astro.it \\ 2 Astrophysikalisches Institut Potsdam, An der Sternwarte 16, 14482 Potsdam, Germany \\ e-mail: deschoenberner@aip.de,msteffen@aip.de \\ 3 CINECA Interuniversity Consortium, via Magnanelli 6/3, 40033 Casalecchio di Reno (BO), Italy \\ e-mail: c.calonaci@cineca.it
}

Received 28 July 2003 / Accepted 24 October 2003

\begin{abstract}
We follow hydrodynamically the evolution of spherical model planetary nebulae subject to different initial conditions and with various central stars, investigating how combinations of central-star mass and asymptotic giant branch mass-loss rate determine the shape and kinematics of a planetary nebula. With this approach we aim at constituting a framework useful for the interpretation of the evolutionary status and previous mass-loss history of observed individual nebulae, making use of their kinematical properties and surface brightness characteristics. In particular, the models are compared with the observed morphologies and kinematics of double shell nebulae. The dynamical structure of all the models is characterized by a more or less complicated shock wave pattern set up by ionization and wind interaction whose combined action results in general in a typical double-shell structure. We have found that models with simple initial structures based on a constant AGB massloss rate fail to comply with observed shell morphologies and surface-brightness distributions. A reasonable agreement with the observations is only found for a model where the mass-loss rate is strongly increasing towards the end of the asymptotic giant-branch evolution. Depending on the central star's evolutionary speed and the density of the cool wind expelled along the asymptotic giant-branch, planetary nebulae may never get optically thin. This is primarily the case for the more massive central stars, and this fact offers a rather natural explanation for the long standing problem of the very existence of molecular hydrogen in the immediate vicinity of hot central stars. We also show that distances to planetary nebulae based on expansion parallaxes are systematically too small by a significant amount.
\end{abstract}

Key words. hydrodynamics - radiative transfer - planetary nebulae: general - stars: AGB and post-AGB

\section{Introduction}

The importance of a stellar wind to explain the basic morphological aspect of a typical Planetary Nebula (PN) consisting of a bright ring/shell with a clear border on its inner side and a significant reduction of surface brightness between this border and the central star (CSPN), was first advocated by Mathews (1966). This idea was later developed by Kwok et al. (1978) in the so called ISW (Interacting Stellar Wind) theory. Just at that time the IUE satellite started to prove the existence of fast stellar winds as a common phenomenon also for CSPN (Heap et al. 1978). The properties of these winds where then extensively studied both observationally (e.g. Cerruti-Sola \& Perinotto 1985; Perinotto 1993; Patriarchi \& Perinotto 1991) and theoretically (Pauldrach et al. 1988; Kudritzki et al. 1997;

Send offprint requests to: M. Perinotto, e-mail: Msteffen@aip. de
Kudritzki 1998). It became then clear that the paradigm by Mathews correctly applies to real objects.

An important improvement of the original ISW theory was given by Giuliani (1981) who included the dynamical influence of ionization fronts and calculated the variations of density and velocity within the flow by use of similarity transformations. He showed that the expanding shell has always a positive velocity gradient, and that a PN may not become optically thin for massive central stars which fade sufficiently fast.

The early hydrodynamical studies, like that of Mathews mentioned above, were based on the "explosive" ejection, i.e. a gaseous shell with given density and velocity profile was allowed to expand into virtually empty space (Sofia \& Hunter 1968; Ferch \& Salpeter 1975). Ferch \& Salpeter included a dust component and were thus also able to avoid the infall of matter without invoking support by a stellar wind. Later on various authors have then approached the problem of following the 
morphological development of a PN within the frame of the ISW theory mentioned above, viz. by considering the interaction between the fast and tenuous wind from the central star with the slow and dense one produced earlier when the star was still on the asymptotic giant branch (AGB).

With particular attention to detailed modeling one has to mention the work of two groups. Relevant papers of the first group include those by Icke (1988, 1991), Mellema et al. (1991), Icke et al. (1992), Frank \& Mellema (1994a,b), Frank (1994), Mellema (1994, 1995, 1997), and Mellema \& Frank (1995). Relevant papers by the second group are those by Marten \& Schönberner (1991), Schönberner et al. (1997), Marten \& Szczerba (1997), Perinotto et al. (1998), and Steffen et al. (1998).

Other important contributions to gas dynamics in PNe include those by Schmidt-Voigt \& Köppen (1987a, 1987b), Kahn \& Breitschwerdt (1990), Breitschwerdt \& Kahn (1990) and García-Segura et al. (1999). The latter authors emphasized the importance of magnetic fields, if present, for the shaping of (axisymmetric) PNe. The Okorokov et al. (1985) study is the only one that treated the winds as consisting of two fluids, one being the gas and the other being the dust component, coupled by drag. In this respect it should be mentioned that all the studies quoted here do not include dust if not otherwise stated.

The paper of Bobrowsky \& Zipoy (1989) deserves a special comment despite the fact that it is based on the "explosive" scenario. It is the only study assuming the initial envelope to consist entirely of molecular hydrogen. The dissociation of molecular hydrogen during the evolution is carefully treated, but the outcome is of only rather limited use for interpreting observations since the models are not very realistic from a modern point of view.

All these previous studies have clarified that the basic idea of the ISW concept, if ionization is taken into account, can conceivably reproduce what broadly speaking is "seen" observationally in $\mathrm{PNe}$, at least in round $(\mathrm{R})$ or moderately elliptical (E) PNe. This excludes especially the so-called bipolar PNe, where more complex processes have to be invoked, as aspherical winds induced by various mechanisms, or magnetic fields, for instance, but where an unique paradigm based on first principles is still lacking.

It is now widely accepted that PN evolution in $\mathrm{R} / \mathrm{E}$ objects is dominated by i) the AGB mass-loss history and ii) evolution of the remnant star with its changing ionizing flux and wind power. At present it is, however, still unknown how the mutual influence of i) with ii) affects the structure and kinematics of an individual PN, in particular how it causes a) the often observed positive velocity gradients, $b$ ) the formation of doubleshell structures with the observed relative size and velocities of the respective shells, c) the very existence of neutral matter with a temperature sufficient to excite molecular transitions, and d) the existence of more than one halo.

To understand these various aspects we think that the proper method requires first to construct a grid of detailed models able to follow the hydrodynamical evolutionary behaviour of a PN from the formation to its final phases. This grid should help to understand the general properties mentioned under a), b), c), and d) above, and it will also assist with the more ambitious purpose of correctly reproducing individual $\mathrm{PNe}$, with the final goal to fully understand their nature, their present evolutionary phase, and to be able to infer their entire past history from the main sequence and to predict their evolution to the white-dwarf domain as well.

The only parameter studies based on radiationhydrodynamics simulations so far existing are those of Schmidt-Voigt \& Köppen (1987b) and Mellema (1994, 1995). Our study contains, however, a larger set of initial models, of associated central-star evolutionary tracks, and covers a much longer evolutionary time span, reaching from the tip of the AGB to the realm of hot white dwarfs.

Villaver et al. (2002a,b) studied the dynamical evolution of circumstellar gas envelopes around AGB and post-AGB stars. Their approach is based on the evolutionary models of Vassiliadis \& Wood (1993, 1994) which include empirically determined parametrizations of mass-loss rate and (terminal) outflow velocity as a function of the model parameters. Using the prescribed values for mass loss and outflow velocity, Villaver et al. (2002b) computed the response of the (spherical) circumstellar gas envelope as the post-AGB star evolves. There are several assumption made to simplify the physical processes involved:

i) radiation transfer is not included, with the consequence that ionization is treated in the Strömgren approximation, i.e. the radial distance of the ionization front is determined by assuming that ionization equilibrium holds all the time and that ionization is complete within the $\mathrm{H}$ II region. This is a rather poor approximation when densities become smaller and recombination time scales become comparable to the evolutionary ones (cf. Marten 1995).

ii) Within the ionized region, the (electron) temperature is set to the fixed value of $10000 \mathrm{~K}$, whereas under more realistic conditions this temperature may vary between 5000 and $15000 \mathrm{~K}$, depending on the effective temperature of the central star.

iii) The (transition) time needed for an AGB remnant to reach the PN domain is neglected. Specifically, the authors assume that the post-AGB model starts with $T_{\text {eff }}=10000 \mathrm{~K}$ immediately after the AGB phase is finished, loosing thereby consistency with the evolutionary calculations.

Marigo et al. (2002) developed a method to describe the evolution of planetary nebulae by a so-called "synthetic" model. The advantage of such an approach is that global observable quantities and their dependences on input parameters can be computed in a very fast and efficient way. One can not, however, get insight into the delicate interplay between ionization and wind interaction which are responsible for the evolution of the morphology and the velocity field with time (cf. Schönberner et al. 1997; Schönberner \& Steffen 2003a).

With the present paper we i) illustrate our efforts done during the last years to calculate the above mentioned grid of theoretical evolutionary "tracks" of model PNe by means of detailed radiation-hydrodynamics calculations in a fully timedependent manner, ii) discuss the basic behaviour of these "tracks" and iii) confront them with real PNe. In Sect. 2 details of the physical model and its numerical treatment are given. The calculated evolutionary tracks are summarized in Sect. 3 and explained in more detail in Sect. 4. Some general results 


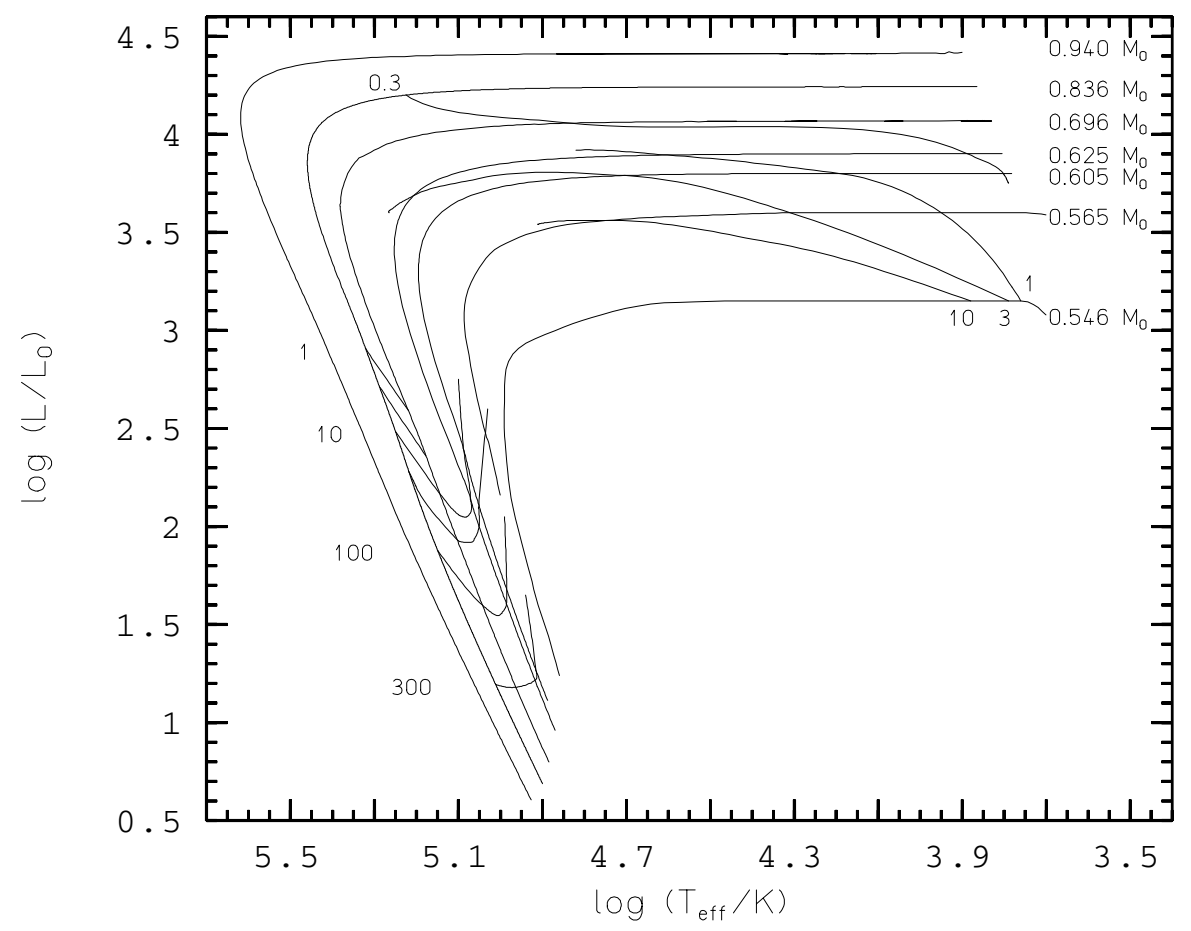

Fig. 1. The Hertzsprung-Russell diagram with the evolutionary tracks of hydrogen-burning AGB remnants, computed consistently by Blöcker (1995b) for different initial masses $\left(3 M_{\odot}\right.$ to $7 M_{\odot}$ ) through all relevant phases including mass loss. The two lowest-mass tracks $(0.565$ and $0.546 M_{\odot}$ ) are from Schönberner (1983) and have initial masses of 1 and $0.8 M_{\odot}$, resp. The isochrones are labeled in units of $10^{3}$ years, and the zero points of the post-AGB ages are at the beginnings of the tracks in the right upper corner of the diagram. They correspond to the (fundamental) radial pulsation period of 50 days for the Blöcker models, and to $T_{\text {eff }}=5000 \mathrm{~K}$ for the Schönberner models.

of our simulations are discussed in Sect. 5, while a comparison with observations is given in Sect. 6. The article is concluded with Sect. 7.

\section{The hydrodynamical models}

In order to describe satisfactorily the evolution of a model nebula from the formation at the tip of the AGB towards the final phases, one should i) precisely fix its "initial conditions", which imply a knowledge of the so-called slow wind, i.e. the conspicuous mass ejection occurring during the last AGB phases, ii) provide an adequate description of the subsequent 'fast wind', and iii) follow hydrodynamically the interaction of both winds under the influence of the ever changing radiation field of the evolving central star.

\subsection{The central-star models}

Since it is the central star that drives the formation and evolution of a PN, points ii) and iii) call for a specification of the post-AGB models to be employed. We use the post-AGB evolutionary models of Blöcker (1995b) and Schönberner (1983) which have the advantage to cover a very wide range of remnant masses, from 0.546 to $0.94 M_{\odot}$. The tracks are illustrated and explained in Fig. 1. Isochrones indicate the strong dependence of the evolutionary speed across the Hertzsprung-Russell diagram on remnant mass and include the transition phases as they result from the computation for the given post-AGB mass-loss prescription (see also discussion in Schönberner \& Blöcker (1993). We note that AGB remnants with masses around $0.6 M_{\odot}$ - with fading times of about 10000 years are most relevant for investigating planetary nebula evolution.

The post-AGB tracks shown in Fig. 1 are burning hydrogen as their main energy supply and should be typical for the majority of the central stars with normal surface compositions (cf. Schönberner 1981; Corradi et al. 2003). Central stars with Wolf-Rayet spectra are definitively hydrogen depleted and must burn helium. Their creation is most likely due to the occurrence of a thermal pulse during the transit towards the white-dwarf domain. The pulse drives strong convection which mixes and burns hydrogen very rapidly on a convective turnover time scale (cf. Herwig 2001; Blöcker 2001). Since very little is known about the temporal evolution of the WR central stars, they are not considered in our study. Likewise, we do not consider models where the thermal pulse occurs deep inside the PN region and forces the model to return to the vicinity of the AGB for a short time, although our radiationhydrodynamics code is able to deal with even the fastest conceivable evolutionary speed of a central star.

\subsection{The AGB wind}

Point i) above requires a knowledge of the behaviour of the progenitor star during the final AGB evolution, especially the details of the mass-loss history. This is a major problem, and at present this AGB mass-loss history can only be estimated. For the purpose of this work, we used three approximations, 
intended to reproduce the density and velocity distribution of the expanding material surrounding the central star by the time it has reached the end of the AGB evolutionary phase. They provide the initial conditions for the following hydrodynamical evolutionary sequences.

TYPE A: In this rather simple case the density distribution of the AGB wind, $\rho(r)$, is based on constant values of mass loss rate, $\dot{M}_{\mathrm{agb}}$, and terminal wind velocity, $V_{\mathrm{agb}}$, resulting in a $\rho \propto$ $r^{-2}$ law. The inner boundary is set at a distance of $1 \times 10^{15} \mathrm{~cm}$ from the star, where the mass loss is assumed to drop virtually instantaneously from $\dot{M}_{\mathrm{agb}}$ to the Reimers' rate (Reimers 1975) at $t=0$.

TYPE B: A second, better approach is to allow for mass-loss variations but keeping the wind speed constant. Hydrodynamical simulations along the upper AGB suggest that, despite a significant mass-loss variation, the outflow velocity varies only very modestly, as can be seen in Fig. 2 (bottom panel). Fixing the AGB-wind speed leads then to density structures that reflect rather directly the time dependent AGB mass-loss rate and are thus certainly more realistic than assuming constant outflows, without invoking time-consuming radiation-hydrodynamics simulations. The remaining problem, however, is the choice of an appropriate outflow velocity.

Here the mass loss is taken from the prescription of Blöcker (1995a, Fig. 7), and an outflow velocity of $10 \mathrm{~km} \mathrm{~s}^{-1}$ has been chosen. This is a purely kinematical approach, and these models may thus be called "kinematical" models.

TYPE C: Finally, the density structure and velocity field is derived from detailed radiation-hydrodynamics simulations along the upper AGB (Schönberner et al. 1997; Steffen et al. 1998). Also these models are based on the AGB mass-loss prescription by Blöcker (1995a). We call these models "hydrodynamical" models. They can be considered as the most consistent nebular models calculated so far. Although they are based on a two-fluid dust-gas code, they serve here as input for a gas-only code used to follow the post-AGB evolution (see Sect. 2.4).

In all AGB wind models we assume a chemical composition typical for planetary nebulae (see Perinotto et al. 1998). The envelope is initially neutral with a temperature of $100 \mathrm{~K}$. Molecules are not considered.

In order to get a complete starting model, we combined the different types of initial envelopes with appropriate post-AGB models of age zero. Illustrative examples of the three kinds of initial configurations considered here are given in Fig. 2.

The differences between the models of TYPE A and those of B and C are quite obvious: for the two latter cases, i.e. the "kinematical" and "hydrodynamical" approaches, the initial structures depend on the previous mass loss history, and their radial density distributions differ considerably from the simple law for constant flow properties. Moreover, they are also heavily modulated by the last thermal pulse(s) during the final AGB evolution (cf. Blöcker 1995a, Fig. 7). In particular, we found $\rho \propto r^{-1} \ldots r^{-2.3}$ for $M=0.625 M_{\odot}$, and $\rho \propto r^{-2} \ldots r^{-2.5}$ for $M=0.696 M_{\odot}$, depending on the radial position. It should be

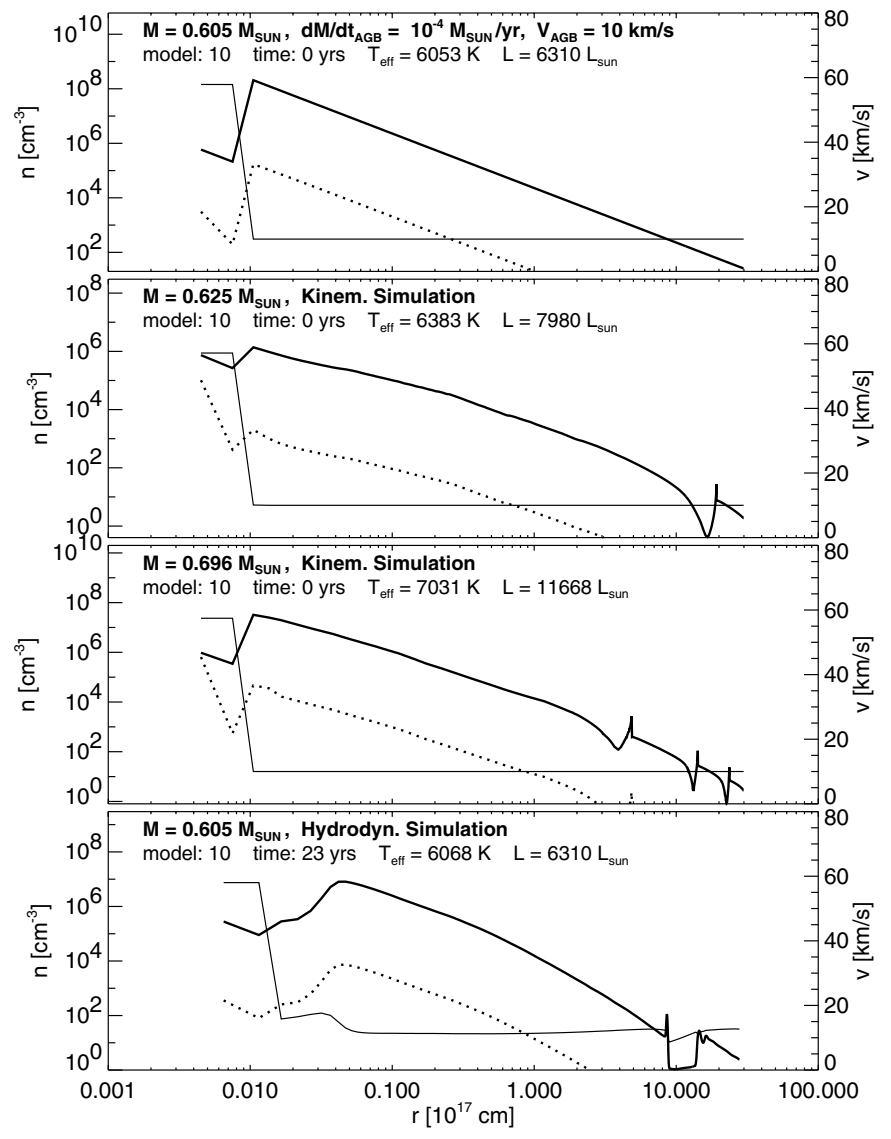

Fig. 2. Initial AGB wind-envelope structures: heavy particle densities (thick), electron densities (dotted), and flow velocities (thin) as a function of radius. Envelope and stellar parameters are given in the individual panels. Top: example of an AGB model with constant massloss rate and wind speed (TYPE A). Middle panels: density structures caused by mass-loss variations close to the tip of the AGB, assuming a constant wind speed of $10 \mathrm{~km} \mathrm{~s}^{-1}$ (see Blöcker 1995a, Fig. 7 therein, and Calonaci 2000 for details). The density modulations are signatures of the last thermal pulses on the AGB (TYPE B). Bottom: density and velocity profiles resulting from full hydrodynamical simulations along the upper AGB (Steffen et al. 1998). The large density trough at $\sim 10^{18} \mathrm{~cm}$ is due to the last thermal pulse 40000 years before the star left the AGB (TYPE C).

noted that the "hydrodynamical" model shows an even larger density gradient further out $\left(\rho \propto r^{-3.5}\right)$.

All these steeper density gradients are caused by the strong mass-loss increase towards the end of the AGB evolution mainly due to the $M^{-2.1}$ dependence of the mass-loss description employed by Blöcker $(1995 \mathrm{a})^{1}$. Moreover, the main circumstellar shell of the TYPE $\mathrm{C}$ model is, beginning at $\approx 10^{18} \mathrm{~cm}$, surrounded by an outer low-density region which is the result of the last thermal pulse and which separates matter that has been expelled earlier. More details about the properties of this model can be found in Steffen et al. (1998).

The mass-loss rates used for constructing the initial models appear rather high compared to what is typically known from

1 The latest compilation of wind envelope models for carbon-rich compositions indicate a similar dependence of the mass-loss rate on the actual stellar mass: $\dot{M} \propto M^{-1.95}$ (Wachter et al. 2002). 

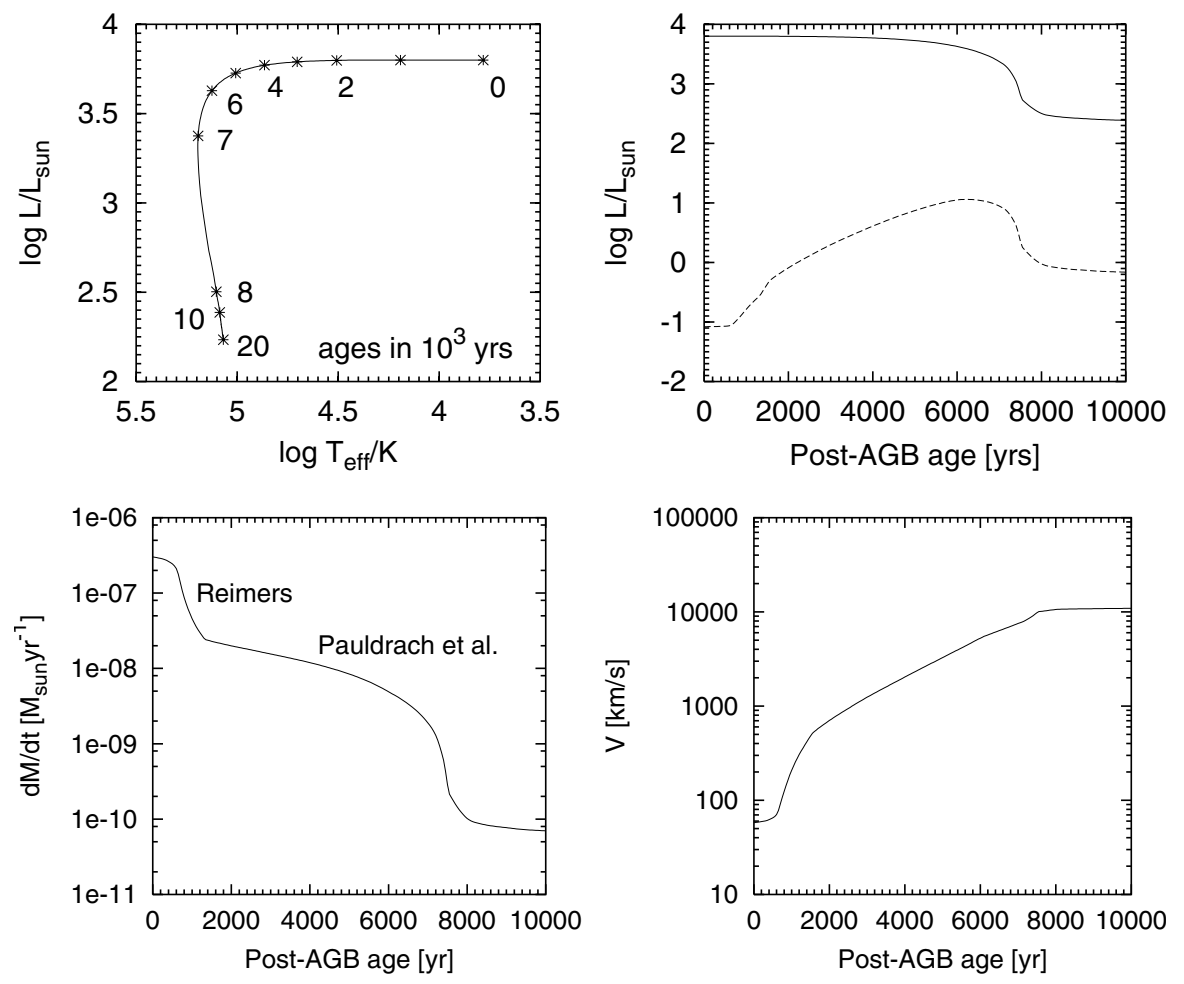

Fig. 3. Top panels: evolutionary path for the $0.605 M_{\odot}$ post-AGB model with ages indicated (left), and the corresponding photon and wind luminosity, $\dot{M} \cdot V^{2} / 2$ (dashed line) vs. time (right). Bottom panels: mass-loss rate (left) and wind velocity (right).

observation of mass-loosing AGB stars. One has, however, to consider that we are dealing with the very short-lived end phase of the AGB evolution, and a better estimate of the corresponding mass-loss rates is gained by evaluating the emission from detached dust shells of post-AGB objects: final AGB mass-loss rates vary from $\simeq 10^{-5}$ up to above $10^{-4} M_{\odot} /$ yr (Hrivnak et al. 1989; Bujarrabal et al. 2001). Observations also suggest that these very high rates continue until the central object becomes rather hot, say $5000 \mathrm{~K}$ or more, and drops then to rather low values within a few 100 years (cf. Schönberner \& Blöcker 1993; Fuji et al. 2001). As an example, we refer to the recent determination of the properties of the circumstellar shell around the post-AGB star HD 161796 with an effective temperature of $6750 \mathrm{~K}$ : the mass outflow with a rate of $\dot{M}=5 \times 10^{-4} M_{\odot} / \mathrm{yr}$ and a speed of $15 \mathrm{~km} \mathrm{~s}^{-1}$ ended only 430 years ago (Hoogzaad et al. 2002).

In the simplest models without a detailed mass-loss description, i.e. those with TYPES A envelopes, we kept the assumed AGB rate up to the starting point of the post-AGB evolution.

\subsection{The central-star wind}

As with the fast wind central-star wind, the theory of radiationdriven winds as formulated by Kudritzki and collaborators is considered to correctly represent the main physical mechanism, given its success for hot Pop. I stars of high luminosity (cf. Kudritzki \& Puls 1999). We thus computed the properties of the fast wind, i.e. mass-loss rate and wind speed, as outlined by Kudritzki et al. (1989) with the line-force parameters recommended by Pauldrach et al. (1988). Below about $25000 \mathrm{~K}$ where the theory of radiation-driven winds is not applicable we used the interpolation described in Marten \& Schönberner (1991). Specifically, the mass-loss rate of Reimers (1975) is used for this purpose. Fortunately, the dynamical impact of the stellar wind is rather small during this transition phase, making a more detailed description not really necessary (see Schönberner \& Steffen 2001).

We note that the speed of the central-star wind is close to $60 \mathrm{~km} \mathrm{~s}^{-1}$ at the beginning of the post-AGB evolution, but increases during the course of evolution to approx. $10000 \mathrm{~km} \mathrm{~s}^{-1}$ when white-dwarf configurations are reached. We also emphasize that the mass-loss rates used here are in full agreement with those used by Blöcker (1995b), thus avoiding any inconsistencies between nebular and stellar evolution. The rates scale with $L^{1.9}$, and for a $0.6 M_{\odot}$ central star we have a mass-loss rate of about $10^{-8} M_{\odot} /$ yr during the high-luminosity phase of evolution, fully consistent with best observational determinations (Perinotto 1993). This is much below the hydrogen burning rate of about $10^{-7} M_{\odot} / \mathrm{yr}$.

The physical properties of the post-AGB wind as assumed in the present work are illustrated in Fig. 3 for the $0.605 M_{\odot}$ model. It is important to note that the generally decreasing mass loss is more than counteracted by the increase of the wind velocity: the kinetic energy carried away by the wind, $\dot{M} \cdot V^{2} / 2$, i.e. the wind power, rises substantially until the star reaches the turn-around point in the Hertzsprung-Russell diagram. It remains, however, always negligible compared to the photon luminosity.

The wind properties are similar for other remnant masses: on the horizontal part of evolution where the wind impact is largest, the mass-loss rate increases with mass because of the 
larger (photon) luminosity, while the wind speed decreases because of the smaller escape velocity.

\subsection{The numerical treatment}

The interaction of the fast wind with the slow wind under the changing radiation field of the evolving central star is represented by classical hydrodynamical equations. They are solved in spherical geometry by an explicit technique based on a second-order Gudunov-type advection scheme. The numerical grid contains 1000 mesh points and extends, in most cases, from $5 \times 10^{14}$ to $3 \times 10^{18} \mathrm{~cm}$. Density and velocity at the inner grid boundary are given by the actual values of (mass) density and velocity of the central-star wind.

As with the radiation part of the physical system, it has been shown by Marten $(1993,1995)$ that the evolutionary time scales of central stars may become comparable to the heating/cooling and ionization/recombination time scales of the gas. A fully time-dependent treatment of the ionization and energy balance is thus needed. We used a code developed by Marten \& Sczcerba (1997) which considers time-dependent ionization, recombination, heating and cooling of $\mathrm{H}, \mathrm{He}, \mathrm{C}$, $\mathrm{N}, \mathrm{O}, \mathrm{Ne}$, including all of their ionization stages. The (nonequilibrium) cooling function is computed from all ions involved, using the actual local densities and electron temperatures. Radiative transport makes use of the "on-the-spot" approximation for computing the diffuse radiation field. More details of the numerics used in this work can be found in Perinotto et al. (1998).

The very detailed microphysics implemented in the code that is needed to realistically follow all time-dependent variables during the evolution of the models requires quite long CPU times even on fast modern workstations. To speedup the calculations, an effort has been devoted to parallelize the code (Calonaci et al. 2001). This allowed to efficiently perform calculations on parallel machines of the last generation. Part of the calculations presented in this paper have been done on the IBM SP3 of the Computing Center of Bologna (CINECA).

\section{Calculated evolutionary sequences}

We computed various evolutionary sequences consisting of different combinations of envelope (see Sect. 2.2) and central-star models, in order to provide a framework useful for interpreting the general characteristics of planetary nebulae. All the combinations of central stars and envelopes that have been computed and which are discussed in this work are listed in Table 1. The first column refers to a running sequence number, the second gives the mass of the central star, the third and fourth AGB mass-loss rate (if applicable) and (terminal) AGB wind speed resp., and the fifth characterizes the type of the initial model (TYPES A, B, or C). We did not consider evolutionary sequences with the $0.546 M_{\odot}$ model since its evolution towards the white-dwarf stage exceeds greatly the dissipation time scale of an AGB wind envelope, and the formation of a PN is not possible in this case.
Table 1. Hydrodynamical sequences of model planetary nebulae.

\begin{tabular}{rcccc}
\hline \hline No. & $\begin{array}{c}M \\
M_{\odot}\end{array}$ & $\begin{array}{c}\dot{M}_{\text {agb }} \\
M_{\odot} / \mathrm{yr}\end{array}$ & $\begin{array}{c}V_{\text {agb }} \\
\mathrm{km} \mathrm{s}^{-1}\end{array}$ & TYPE \\
\hline 21 & 0.565 & $1 \times 10^{-5}$ & 10 & $\mathrm{~A}$ \\
22 & 0.565 & $3 \times 10^{-5}$ & 10 & $\mathrm{~A}$ \\
2 & 0.605 & $1 \times 10^{-5}$ & 5 & $\mathrm{~A}$ \\
3 & 0.605 & $3 \times 10^{-5}$ & 10 & $\mathrm{~A}$ \\
4 & 0.605 & $1 \times 10^{-4}$ & 10 & $\mathrm{~A}$ \\
5 & 0.605 & $1 \times 10^{-4}$ & 15 & $\mathrm{~A}$ \\
6 & 0.605 & "Hydro." simulation & & $\mathrm{C}$ \\
7 & 0.625 & "Kinem." simulation & 10 & $\mathrm{~B}$ \\
8 & 0.625 & $1 \times 10^{-4}$ & 15 & $\mathrm{~A}$ \\
9 & 0.696 & "Kinem." simulation & 10 & $\mathrm{~B}$ \\
10 & 0.696 & $1 \times 10^{-4}$ & 15 & $\mathrm{~A}$ \\
11 & 0.836 & $1 \times 10^{-5}$ & 15 & $\mathrm{~A}$ \\
12 & 0.836 & $1 \times 10^{-4}$ & 15 & $\mathrm{~A}$ \\
13 & 0.940 & $1 \times 10^{-5}$ & 15 & $\mathrm{~A}$ \\
14 & 0.940 & $1 \times 10^{-4}$ & 15 & $\mathrm{~A}$ \\
\hline
\end{tabular}

The sequences listed in Table 1 have mainly been selected to address three important aspects of the formation and evolution of planetary nebulae:

1. Different starting models for the same central star mass: sequences Nos. 2, 3, 4, 5 of Table 1.

2. Different central star masses with the same AGB starting model: sequences Nos. 5, 8, 10, 12, 14 of Table 1 .

3. More realistic simulations of the AGB wind: sequences Nos. 6, 7, 9 of Table 1.

We will see that these sequences are sufficient to investigate the basic processes involved in the formation and evolution of PN, i.e. to study how an AGB wind envelope turns into a typical round/elliptical planetary nebula.

\subsection{General evolution}

An example of how typical structures of a PN develop and change with time is given in Fig. 4 for sequence No. 3, a TYPE A initial model with $\dot{M}_{\mathrm{agb}}=3 \times 10^{-5} M_{\odot} / \mathrm{yr}$ and $V_{\mathrm{agb}}=$ $10 \mathrm{~km} \mathrm{~s}^{-1}$, coupled to a $0.605 M_{\odot}$ central-star model. Shown is the logarithm of the normalized density in a position-time diagram, covering the whole computational regime and a total post-AGB evolutionary time span of about 14000 years from the tip of the AGB towards the white-dwarf domain (cf. Fig. 1). This diagram demonstrates nicely how the radial positions of the various fronts (incl. the contact discontinuity) depend on the pressure balance between both sides of the resp. fronts during the course of evolution. In particular, the formation of a bubble of shocked stellar wind matter is seen at age 1000 years when the wind shock detaches from the inner edge of the AGB wind. Another inward "jump" of this "reverse" shock occurs about 6000 years later when the fast wind loses its strength because of the central star's luminosity drop. 


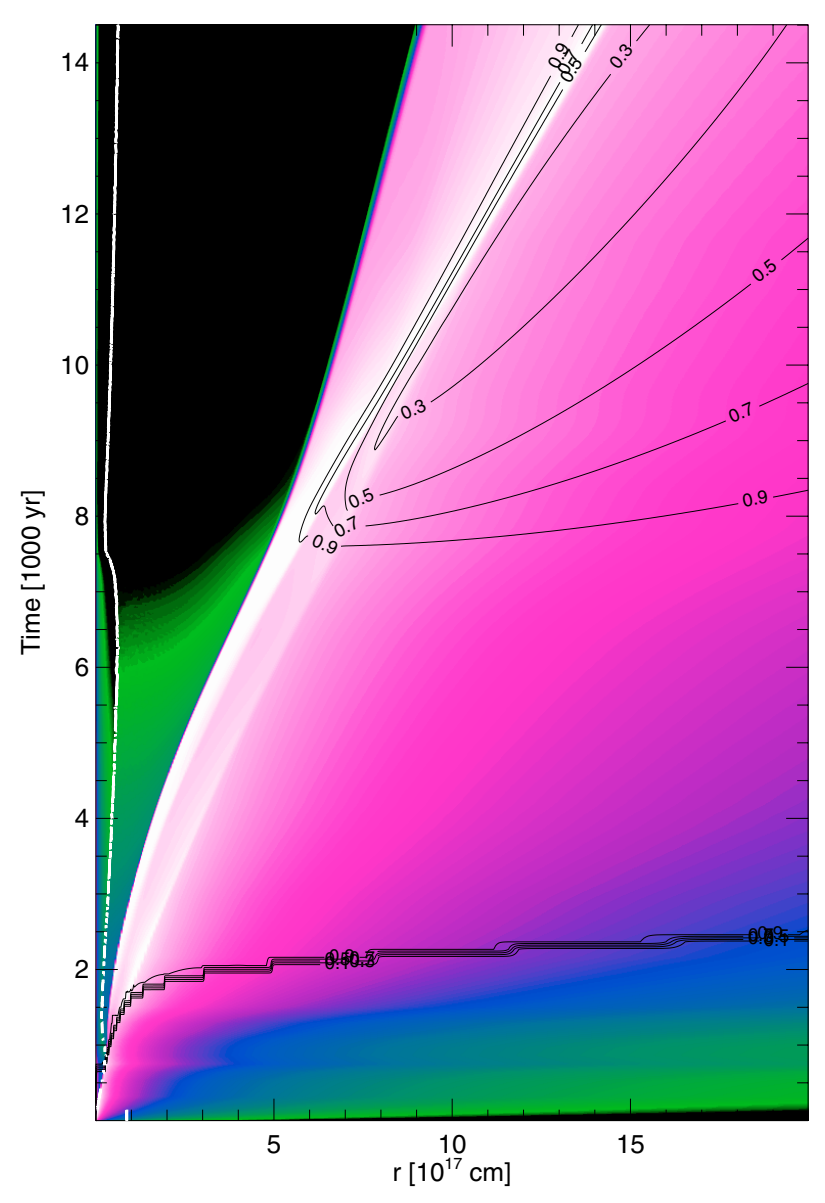

Fig. 4. Position-time diagram for sequence 3 of Table 1 where at each time the logarithm of the normalized density $\rho(r, t) / \rho_{\max }(t)$ is displayed. High-density regions are bright, low-density regions dark. The degree of hydrogen ionization is indicated by the black contour lines. The white line delineates the radial position of the inner shock which thermalizes the central-star wind. The following regions can clearly be distinguished (from right to left): the undisturbed AGB matter, either neutral or ionized (halo), "shell" and "rim" of the planetary nebula proper, the hot, shocked central-star wind (so-called hot bubble), bounded by the inner shock and the contact discontinuity, the latter being the interface which separates the hot bubble from the PN, and the undisturbed central-star wind. See text for a more thorough discussion of the different structures and how they evolve with time.

Figures 5 to 8 illustrate the model structures for selected times indicative of distinguished evolutionary phases along this sequence:

1. The proto-planetary nebula phase where the central star is still too cool to ionize the AGB material. The stellar wind, however, may be ionized, as can be seen in Fig. 5 where the ionization front is trapped at $0.28 \times 10^{17} \mathrm{~cm}$ by the dense AGB material. In this particular stage only the structure of this post-AGB wind, bounded and shaped by the inner edge of the dense and slow AGB wind, is visible in the light of emission lines.

2. The ionization phase where the intense photon flux from a hot central star ionizes and heats also the AGB matter, thereby creating an $\mathrm{H}$ II region which expands into the neutral undisturbed surrounding material due to its large thermal pressure (Fig. 6): a planetary nebula is born at the interface between the slow, dense AGB wind and the tenuous, fast central-star wind by photoionization of the $A G B$ wind. The ionization front (at $r=0.60 \times 10^{17} \mathrm{~cm}$ ) defines its outer, the contact discontinuity (at $r=0.35 \times 10^{17} \mathrm{~cm}$ ) its inner edge.

The thermal pressure of the $\mathrm{H}$ II region accelerates the matter outwards and decelerates it inwards, thereby creating a typical density and velocity structure with large variations of the respective gradients across the ionized region: from very high positive values immediately behind the outer shock to zero or even negative values occurring at the inner edge by the counteraction of the meanwhile increased thermal pressure of the shocked post-AGB wind material, heated to about $2 \times 10^{6} \mathrm{~K}$ by deposition of the kinetic energy carried by this wind (cf. Fig. 6).

For the time shown in the figure, the flow speed immediately behind the outer shock, $21 \mathrm{~km} \mathrm{~s}^{-1}$, is already well above the AGB wind speed of $10 \mathrm{~km} \mathrm{~s}^{-1}$. The shock itself is traveling outwards with $25 \mathrm{~km} \mathrm{~s}^{-1}$, and with $15 \mathrm{~km} \mathrm{~s}^{-1}$ relative to the neutral AGB wind, which is highly supersonic. At the inner regions the matter is nearly stalling $\left(\simeq 3 \mathrm{~km} \mathrm{~s}^{-1}\right)$. The PN is said to be radiation bounded, i.e. optically thick for ionizing photons. Only about 700 years later the ionization front breaks through the outer shock and turns from a D-type to an R-type mode. The PN is now density bounded and confined between the contact discontinuity and the outer shock (cf. Fig. 4).

3. Figure 7 illustrates two snapshots of the compression phase where, at least in this simulation, all the matter within the grid is ionized and the high pressure of the thermalized central-star wind, the hot bubble, compresses and accelerates the inner parts of the ionized AGB material. The contact discontinuity acquires its largest velocity during this particular phase (cf. Fig. 4).

The planetary nebula itself shows now a typical doubleshell structure, consisting of a less dense outer shell originally formed by ionization and a denser inner shell created indirectly by the stellar wind dynamics, i.e. by the expanding contact surface. Morphologically, we identify the outer one with the "shell", the inner one with the bright "rim", following thereby the terminology of Frank et al. (1990). Chu et al. (1987) used the term "attached shell" instead of "shell",

Both shells are preceded by shocks and have quite distinct radial density and velocity profiles: The "shell" has a positive density and velocity gradient, the "rim" generally negative ones. The expansion behaviour of both shells can easily be followed in Fig. 4 and is determined by the pressure balance for the respective shock fronts: The front of the "shell" is driven by thermal pressure against the AGB-wind ram pressure, that of the "rim" is driven against the flow in the "shell" by the (thermal) pressure of the "rim" matter, being actually swept-up "shell" material.

\footnotetext{
${ }^{2}$ Mellema (1995) used the terms "I-shell" and "W-shell" instead of "shell" and "rim", as to indicate that the "shell" is the result of ionization and the "rim" the result of wind interaction.
} 

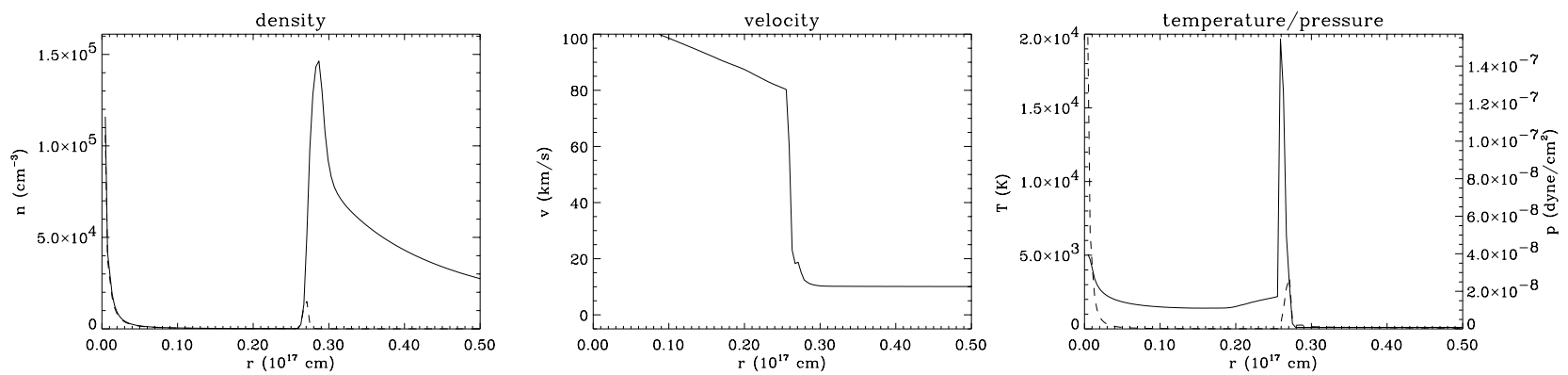

Fig. 5. Radial run of heavy particle density (solid), electron density (dashed), velocity, temperature (solid) and (thermal) pressure (dashed) for sequence No. 3 at $t=748 \mathrm{yr}$, with stellar parameters of $T_{\text {eff }}=10051 \mathrm{~K}$ and $L=6310 L_{\odot}$ (proto-planetary nebula stage).
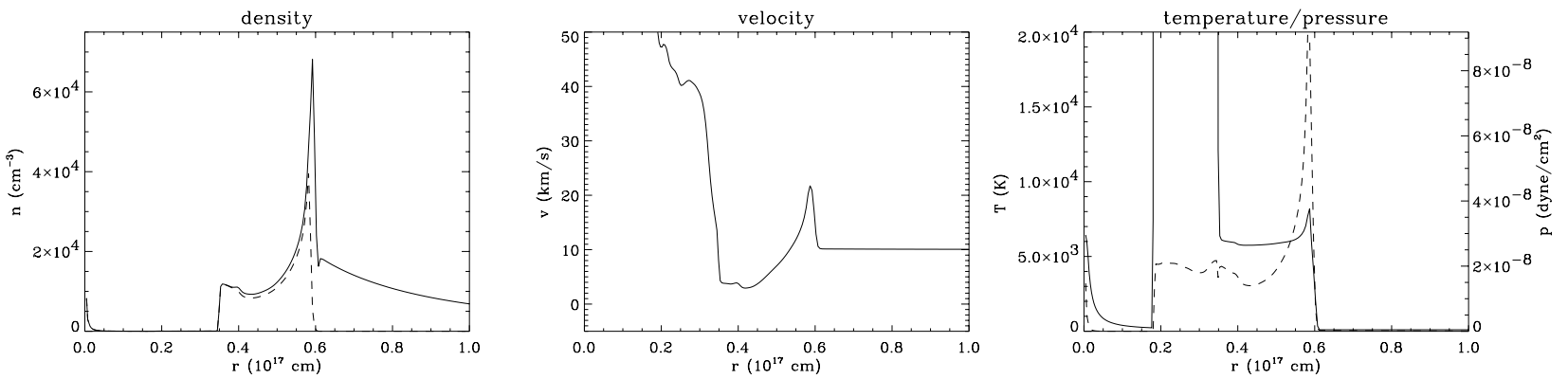

Fig. 6. Same as in Fig. 5 but for $t=1286 \mathrm{yr}$, and $T_{\text {eff }}=20681 \mathrm{~K}, L=6310 L_{\odot}$ (ionization phase). The very thin shell of shocked central-star wind material with $\simeq 20000 \mathrm{~K}$ shown in Fig. 5 at $0.26 \times 10^{17} \mathrm{~cm}$ has changed now to a more extended hot bubble with $\simeq 2 \times 10^{6} \mathrm{~K}$.
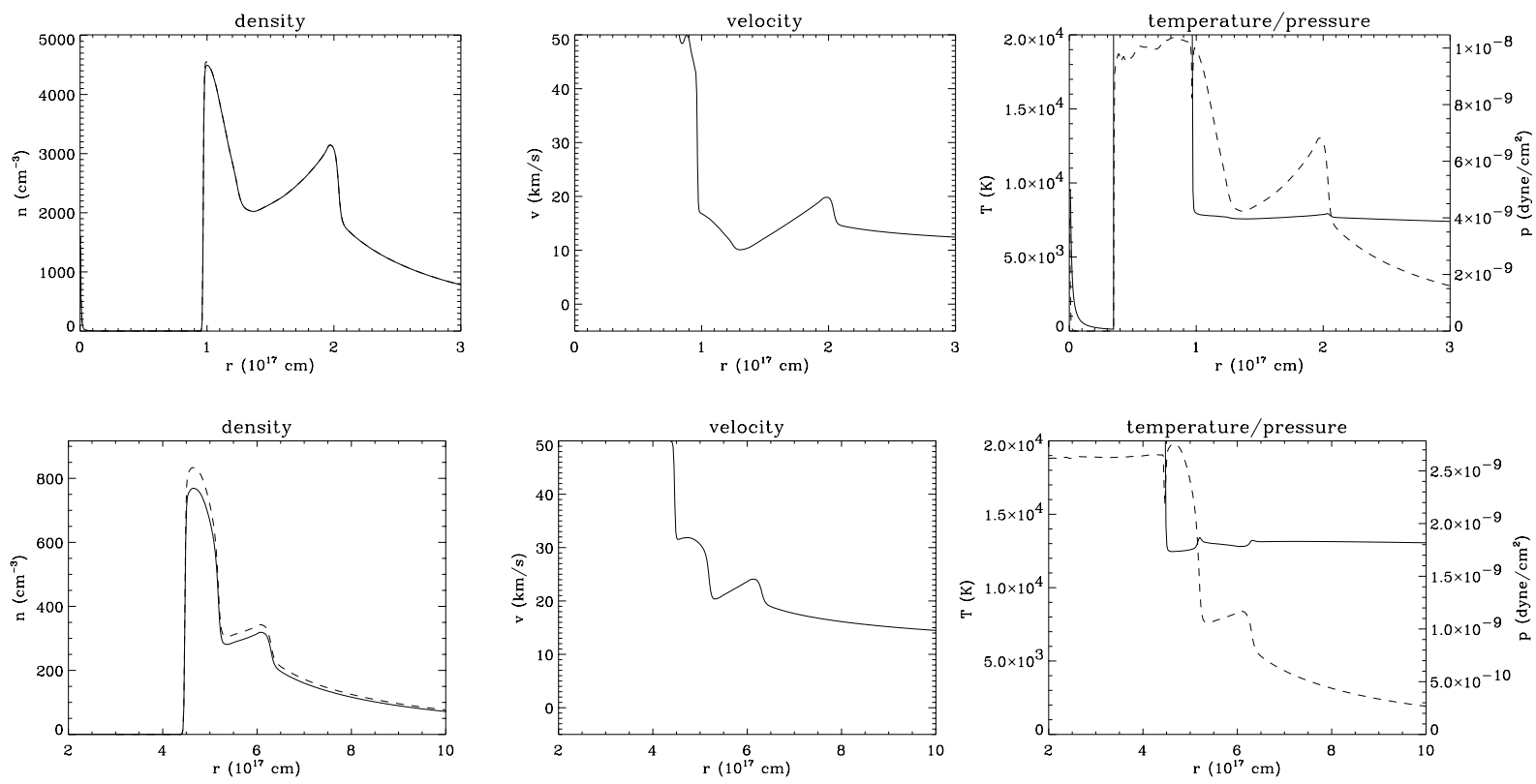

Fig. 7. Same as in Fig. 5 but for $t=2939 \mathrm{yr}, T_{\text {eff }}=49225 \mathrm{~K}, L=6177 L_{\odot}$ (top), and $t=7138 \mathrm{yr}, T_{\text {eff }}=156675 \mathrm{~K}, L=2038 L_{\odot}($ bottom), at maximum effective temperature of the track (compression phase). Note the different radial coverage of the top and bottom panels.

The AGB flow itself is also accelerated by its (thermal) pressure gradient after the gas is heated by ionization and the originally large Mach number thereby substantially reduced. This effect can easily be seen upon comparison of Fig. 6 with both panels of Fig. 7: from the initial speed of $10 \mathrm{~km} \mathrm{~s}^{-1}$ the AGB wind is accelerated to about $20 \mathrm{~km} \mathrm{~s}^{-1}$. For the "shell" it is this increasing speed of the ionized upstream AGB wind that slowly accelerates its front, and the post-shock gas accordingly, whereas in the case of the "rim" it is the increasing pressure behind the front caused by the pile-up of matter due to the rapidly expanding contact discontinuity.

Both shells expand thus independently from each other, and the the front of the "rim" may well overtake that of the "shell" in the course of evolution: in particular, during the compression phase of the simulation shown in 

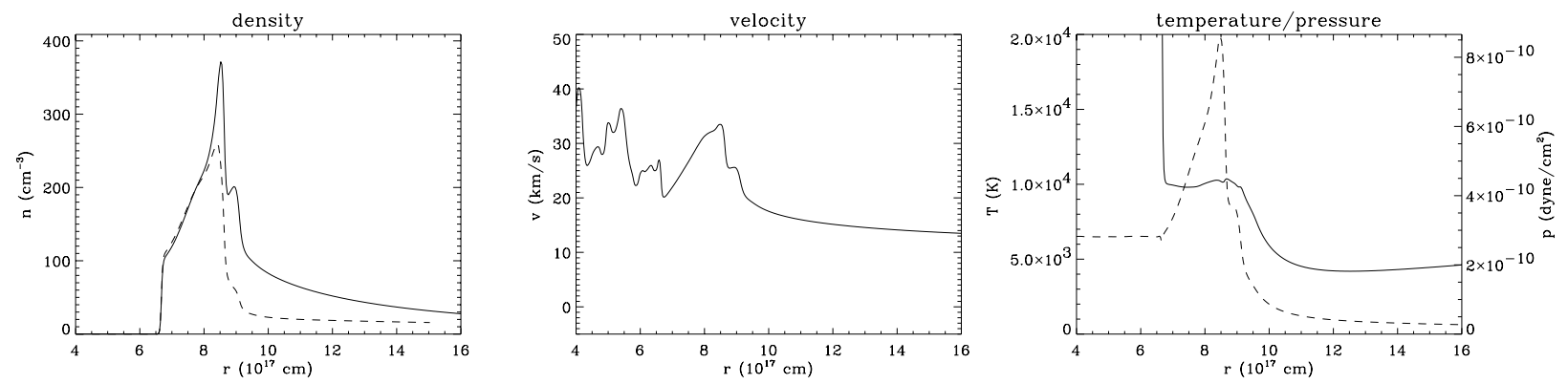

Fig. 8. Same as in Fig. 5 but for $t=9966 \mathrm{yr}$ and $T_{\text {eff }}=122019 \mathrm{~K}, L=244 L_{\odot}$, showing a phase after re-ionization has succeeded in reshaping the inner parts of the PN.

Fig. 4, the shock front of the "shell" reaches a final velocity of $34 \mathrm{~km} \mathrm{~s}^{-1}$, while that of the "rim" has already gained $40 \mathrm{~km} \mathrm{~s}^{-1}$.

In this context it is important to note that the real expansion of a planetary nebula, as seen in projection onto the sky, is given by the speed of this outer shock, and that nowhere within the whole PN structure the flow gains such large values. This disparity between flow velocities and expansion rates is explained by the fact that we are dealing with structures set up by shock waves which compress and accelerate the upstream matter while they expand. The downstream flow velocities are, of course, always lower than the front velocities themselves.

The ionized AGB material surrounding the expanding "shell" constitutes the so-called halo observed around many planetary nebulae. Since the original halo structure will only be slowly changed by ionization, detailed observations of haloes around planetary nebulae may give important clues about a short period of high mass loss occurring immediately before the star leaves the AGB.

The behaviour of the electron temperature within the ionized region deserves a few comments. The steadily increasing surface temperature of the central star is reflected in a corresponding evolution of the electron temperature: starting with a temperature of about $5000 \mathrm{~K}$ at the beginning of the ionization process, the electron temperatures in the nebula reach values as high as $\simeq 13000 \mathrm{~K}$ (cf. Fig. 7, bottom, right) when the central star is hot enough to ionize helium twice. Because of the dominant photo-heating processes throughout the PN evolution, the shocks propagating into the ambient AGB wind are virtually isothermal.

4. Recombination can occur when the central-star evolves rapidly to a white dwarf. Because of the low stellar luminosity the material within the "shell", the outer parts of the "rim", and the halo as well, starts to recombine, provided the time scale of the luminosity drop is sufficiently small compared to the expansion time scale of the circumstellar gas (cf. Tylenda 1986). The degree of recombination depends sensitively on the density of the nebular gas and the fading speed of the central star, and is expected to occur mainly for hydrogen-burning central stars (Schönberner 1981, 1986). For the central-star model of $0.605 M_{\odot}$ discussed here, recombination occurs 7000 years after the end of the AGB. Recombination is also important for more massive central stars with high-density nebular shells which react quickly to the changing conditions of the radiation field (see Sect. 4.1.2).

5. Re-ionization sets in after the fading of the central star has slowed down because of the continued expansion of the circumstellar shells. The bubble's pressure falls behind that of the "rim" because of the small wind power of a whitedwarf central star (cf. Fig. 3), and the contact discontinuity is decelerated accordingly from 30 to $18 \mathrm{~km} \mathrm{~s}^{-1}$ (Fig. 4). The "rim" expands and widens, and its density and velocity structures resemble those typical for ionization, i.e. positive density and velocity gradients develop. The leading shock, however, continues to expand at its high rate and overtakes, finally, the (still partly recombined) "shell".

Figure 8 illustrates a moment during re-ionization when the "shell" is nearly overtaken by the "rim". The recombined areas of the halo and the PN are bounded from within by a new, outward moving ionization front that is driven by the continued expansion. There exists a large gradient in electron temperature between the still recombined and the highly ionized regions: the temperature varies from about $5000 \mathrm{~K}$ in the recombined areas to $10000 \mathrm{~K}$ where full ionization prevails.

\section{Comparison of the different sequences}

To compare the various sequences, we have selected four characteristic phases of the post-AGB evolution: In PHASE I the nebula is still neutral (the so-called proto-PN conditions) with a central star of about $10000 \mathrm{~K}$ effective temperature; in PHASE II the nebula is in general fully ionized with a central star of $T_{\text {eff }} \simeq 50000 \mathrm{~K}$; in PHASE III the central star has reached its maximum temperature along the post-AGB track, and in PHASE IV the luminosity of the star has already decreased to about $250 L_{\odot}$.

Furthermore, we have selected the following combinations of the sequences listed in Table 1: CASE I compares different initial models for the same central-star mass; CASE II deals with the same wind-envelope model for different central-star masses; finally we compare more realistic treatments of the late AGB wind in CASE III. 


\subsection{CASE I: Different starting models for the same central star mass}

\subsection{1. $0.605 M_{\odot}$}

Here we selected four sequences from Table 1 (Nos. 2, 3, 4, 5) with initial envelope of TYPE A but with different massloss rates and outflow velocities. The central-star mass is the same in all cases: $0.605 M_{\odot}$, considered to be the standard or reference mass for a typical central star. According to Blöcker (1995b) an AGB hydrogen-burning remnant of this mass crosses the Hertzsprung-Russell diagram towards the turn-around point in 7000 years and decreases then in luminosity by a factor of seven within only 1000 years when hydrogen burning extinguishes (see Fig. 1).

Phase I: At the particular times shown in Fig. 9, about 750 years after the departure from the tip of the AGB, the central star is still too cool $\left(T_{\text {eff }} \simeq 10000 \mathrm{~K}\right)$ to ionize the rather dense AGB wind, although the central-star wind is already ionized. This figure nicely demonstrates the influence of the (same) central-star wind on AGB envelopes with different densities and speeds: as expected, only the envelope with the lowest density and speed becomes significantly compressed at its inner edge by the wind from the central-star (top frame of Fig. 9).

Phase II: Figure 10 illustrates the situation when the central star is as hot as $\simeq 50000 \mathrm{~K}$. The models from sequences 2,3 , 5 are optically thin and in their compression stage, whereas model No. 4 is still in the ionization stage because of its very high density, and it will in fact never become optically thin. The three optically thin models (No. 2, 3, 5) have developed typical double-shell structures as discussed previously (Sect. 3.1). The relative shell sizes and densities depend on the initial parameters as expected: faster AGB winds create larger "shells", denser AGB winds make denser "shells", as compared with the "rims".

The expansion speeds acquired during the ionization period are quite modest for all three optically thin models: independent of the initial conditions like mass-loss rate and wind velocity, and of the duration of ionization phase, the speeds of the outer shocks have finally settled at $14 \mathrm{~km} \mathrm{~s}^{-1}$ relative to the ambient AGB wind. The density increases across the shock fronts by a factor close to 2 , and the corresponding velocity increase is $5-6 \mathrm{~km} \mathrm{~s}^{-1}$. The maximum flow speeds reached behind the outer shock are between 17 and $23 \mathrm{~km} \mathrm{~s}^{-1}$ for the optically thin cases shown in Fig. 10, depending on the AGB wind velocity. Note that the AGB wind is being self-accelerated by its own pressure gradient once it becomes ionized.

The fact that the expansion properties appear to be rather independent of the initial conditions is astonishing at first glance. In Sect. 5 we will come back to this problem and demonstrate that these properties are explained by means of similarity considerations.

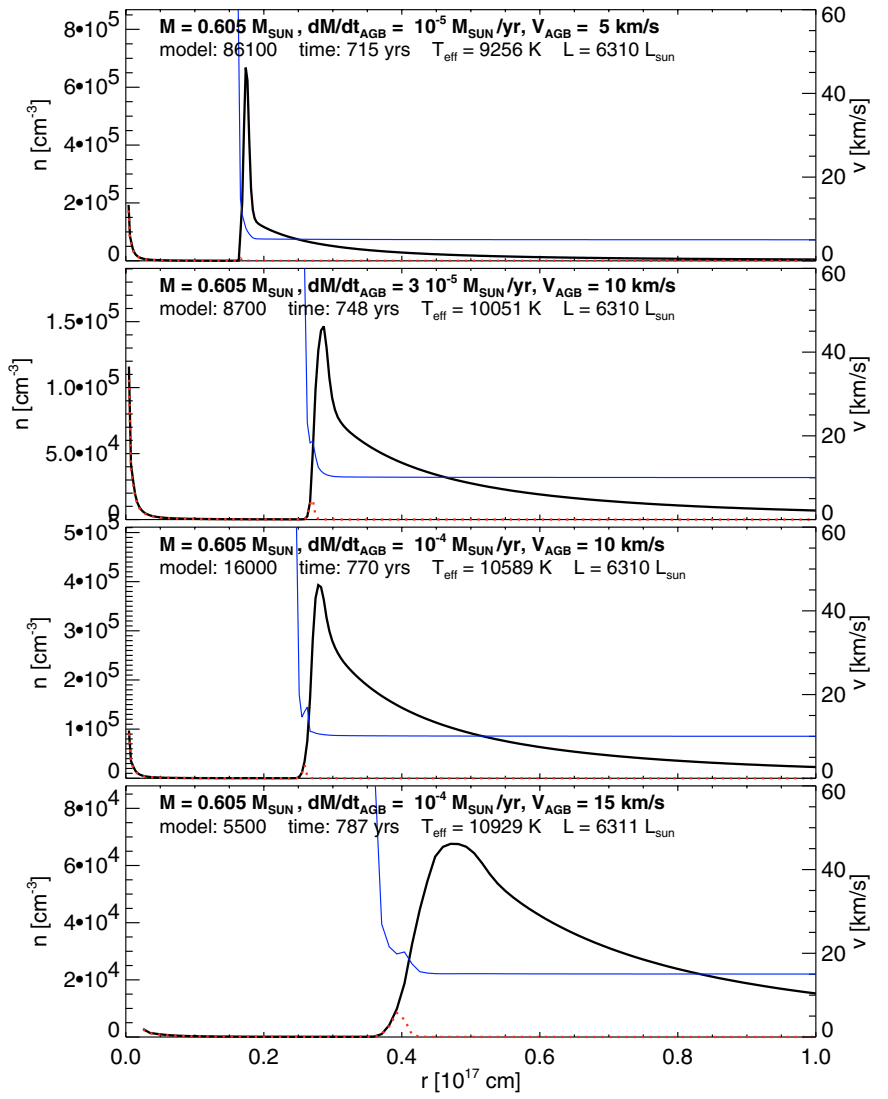

Fig. 9. From top to bottom: heavy particle densities (thick), electron densities (dotted), and flow velocities (thin) for models of the sequences Nos. 2, 3, 4, 5 of Table 1 (CASE I) at PHASE I when the AGB wind envelopes are still neutral while the central-star winds are already ionized. Model and star parameters are given in the individual frames.

PHASE III: Fig. 11 illustrates the situation when the central star has reached its maximum surface temperature before it fades away towards the white-dwarf domain. Now we have maximum compression exerted by the hot bubble: the "rim"s density is now much larger than that in the associated "shell", and, especially in the models with low AGB mass loss rates, the "rim" is about to overtake the "shell" (Fig. 11, top). Note also that the velocity gradient within the "rims" has nearly vanished in all models while that of the "shells" is still positive. The post-shock velocities are now somewhat higher, $22-27 \mathrm{~km} \mathrm{~s}^{-1}$, because the AGB winds have further increased their speed. Especially in the sequences Nos. 2 and 3 the AGB winds have nearly doubled their speed immediately in front of the shock when compared to the initial conditions.

The model of sequence No. 4 deserves a special comment. Because of its very high initial density it did not become optically thin during the high-luminosity phase of evolution. Instead, the outer parts of the $\mathrm{H}$ II region started to recombine already well before the turn-around point. The reduced thermal pressure (decrease of electron temperature and electron number density) decelerates the outer shock front, thereby piling up neutral matter immediately behind the shock and leading to a reverse shock (at $5.3 \times 10^{17} \mathrm{~cm}$ in Fig. 11, panel 3 from top). 


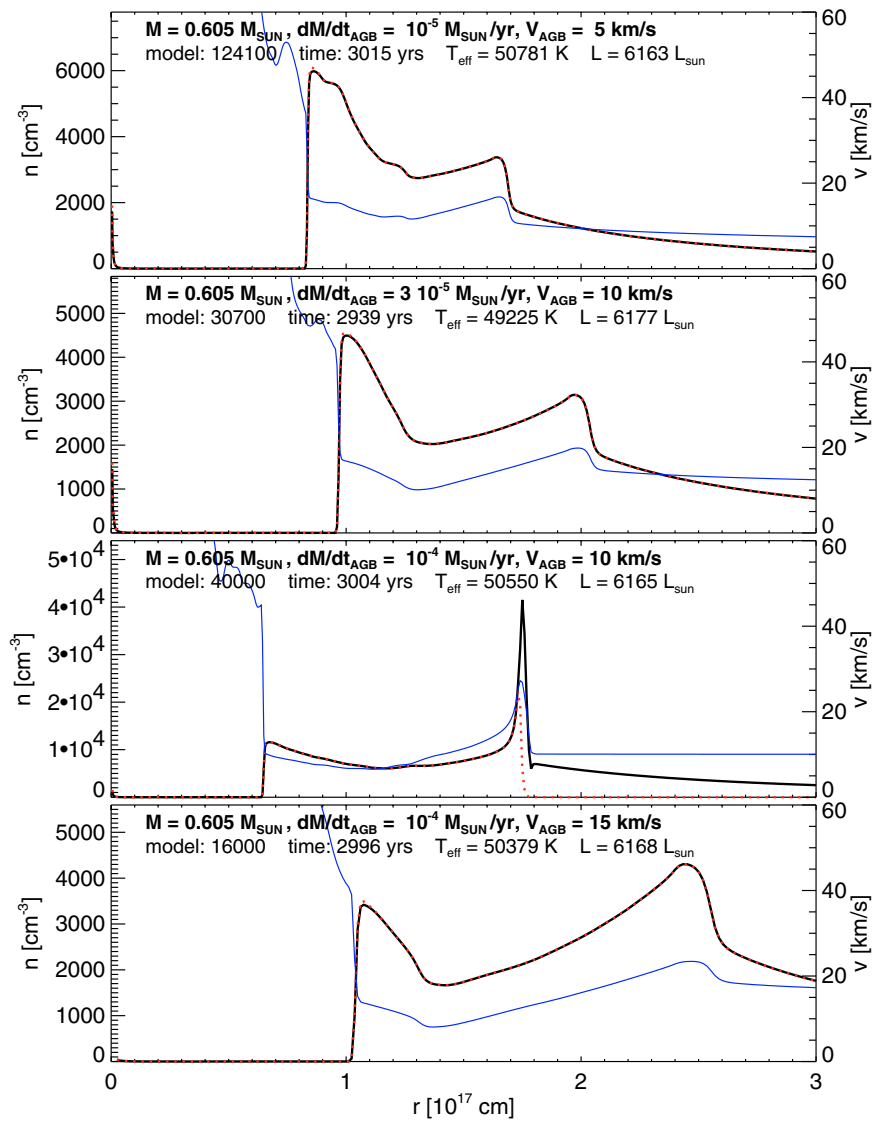

Fig. 10. From top to bottom: models of the sequences Nos. 2, 3, 4, 5 of Table 1 (TYPE I) at PHASE II when the central star is 3000 years old and about $T_{\text {eff }}=50000 \mathrm{~K}$ hot. Now all the matter within the computational regime is fully ionized, except in the model of seq. 4 where the ionization front is still trapped (dashed: electron density). All the optically thin models show well developed double-shell structures. The maximum flow velocities, however, are quite small, around $\simeq 20 \mathrm{~km} \mathrm{~s}^{-1}$.

PHASE IV: Beyond the turn-around point the central-star luminosity drops rapidly, and especially the high density models suffer from recombination because of the reduced ionizing flux (Fig. 12). Only the "rims" remain mainly ionized. Due to the continued expansion, however, re-ionization reshapes them in a similar way as seen in PHASE II, although not as extreme. In all cases the flow velocity increases nearly linearly with radius, with a maximum above $30 \mathrm{~km} \mathrm{~s}^{-1}$ immediately behind the leading edge of the (former) "rims". In the model of sequence No. 2 (top panel of Fig. 12) the "shell" has already completely been swallowed by the fast expanding, reshaped "rim", and the PN morphology has changed accordingly from a double-shell to a single-shell configuration.

When recombination has occurred, one has high ionization in the "rims" and a low one in the "shell", i.e. the PN is highly stratified. Low electron-density regions have also low emissivities, and such a recombined "shell" may be erroneously called a halo. A better term has been coined by Tylenda (1986): recombination halo in order to distinguish them from the ordinary haloes which are simply ionized AGB wind envelopes.

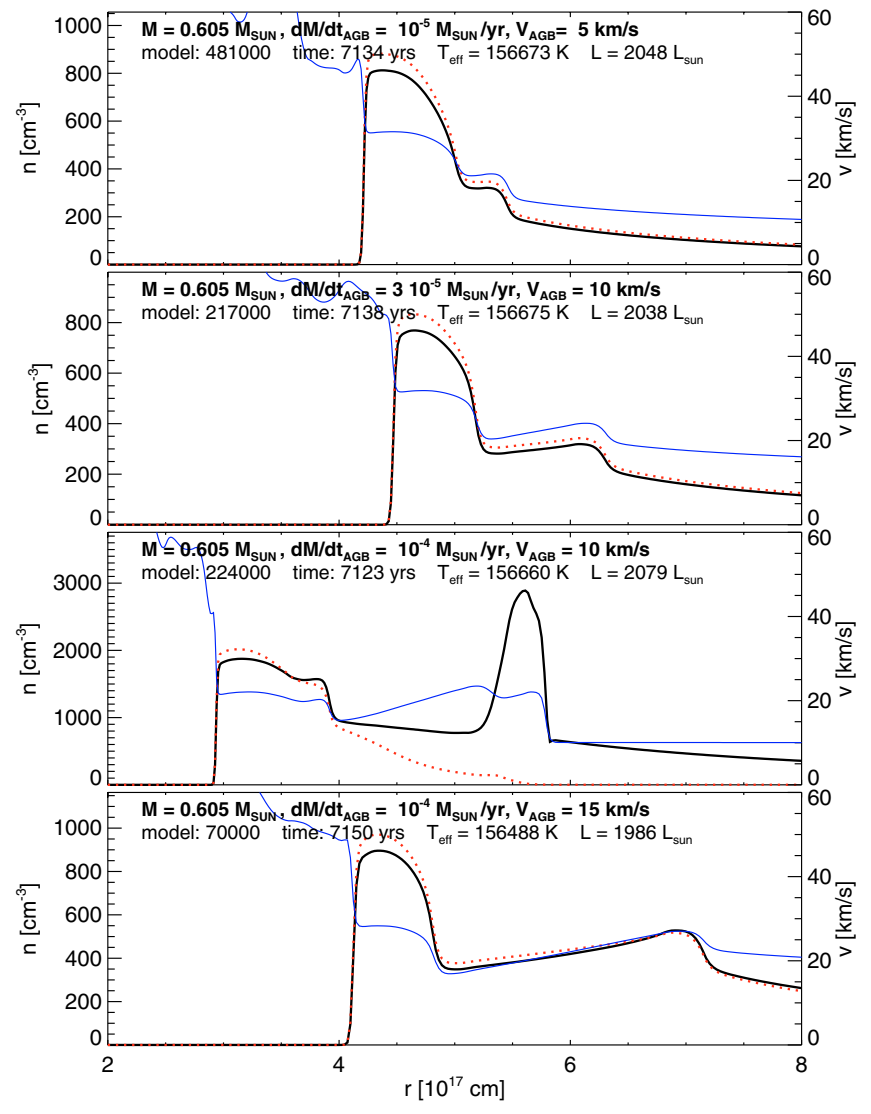

Fig. 11. From top to bottom: models of the sequences Nos. 2, 3, 4, 5 of Table 1 (CASE I) at PHASE III when the central star is at the turnaround point (maximum effective temperatures of $156000 \mathrm{~K}$ ) after 7100 years of post-AGB evolution.

We emphasize that the physical structure of such a recombination halo is quite different from an ordinary AGB halo and is expected to occur only when the central star evolves sufficiently rapidly towards its low-luminosity white-dwarf stage. A recombination halo has recently been identified in NGC 2438 by Corradi et al. (2000).

We noted above that the models of sequence No. 4 never become optically thin. In spite of that, the shock originally set up by ionization (PHASE II) continues to expand into neutral AGB matter and has reached a distance of $8 \times 10^{17} \mathrm{~cm}$ after 10000 years of post-AGB evolution, with a post-shock velocity of $20 \mathrm{~km} \mathrm{~s}^{-1}$ (Fig. 12, panel 3 from top). The neutral matter behind the shock is being compressed and heated up to $3000 \mathrm{~K}$, which is sufficient to excite transitions of molecular hydrogen.

It does not, however, appear to be realistic to assume an AGB mass-loss rate of $10^{-4} M_{\odot} / y r$, lasting for a time span of 10000 years ore more. In this respect the late evolution of sequence No. 4 is of academic interest only. But in the case of more massive central stars which are expected to evolve very quickly to rather low luminosities (cf. Fig. 1), recombination phenomena will be the rule rather than the exception (cf. Sect. 4.1.2). 


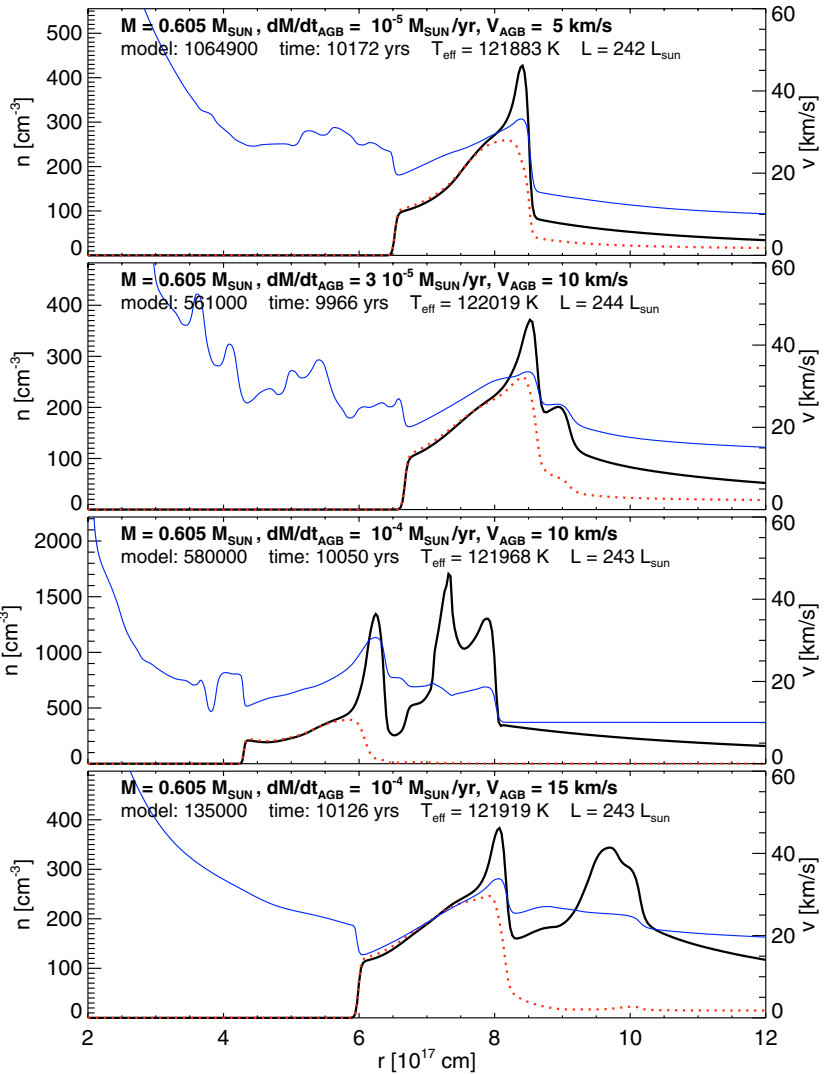

Fig. 12. From top to bottom: models of the sequences Nos. 2, 3, 4, 5 of Table 1 (CASE I) at PHASE IV after about 10000 years when the central-star luminosity has approached the beginning of the whitedwarf cooling path, $L=240 L_{\odot}$.

\subsection{2. $0.836 M_{\odot}$}

The next example illustrates the PN evolution for a massive, very rapidly evolving central star. The $0.836 M_{\odot}$ model reaches the turn-around point in only 400 years after its departure from the AGB, and in additional 300 years it fades by a factor of 10 in luminosity (cf. Fig. 1). The sequences shown in Fig. 13, Nos. 11 and 12 of Table 1, are for envelopes of TYPE A with two different AGB mass-loss rates, $\left(10^{-5}\right.$ and $10^{-4} M_{\odot} / \mathrm{yr}$, resp.), but with the same outflow velocity of $15 \mathrm{~km} \mathrm{~s}^{-1}$.

In both envelope models the intense ionizing radiation field of the luminous central star creates an expanding H II region whose inner part is being compressed into a "rim", as we have already found for the $0.605 M_{\odot}$ sequences. Differences between both $0.836 M_{\odot}$ sequences can be traced back to the initial conditions: the denser model never becomes optically thin during the high-luminosity part of evolution and expands rather slowly, whereas the "rim" of the thinner, fully ionized model acquires such a high velocity that it soon overtakes the shell, leading to a dense, geometrically thin $(\Delta r / r=0.04)$ shell of fully ionized matter with a large velocity gradient and an expansion speed of about $80 \mathrm{~km} \mathrm{~s}^{-1}$ after about 400 years (maximum compression, Fig. 13, middle panel).

Note that during the double-shell phase the (outer) shock has virtually the same properties as in the model sequences No. 2, 3, 5 discussed earlier: the AGB wind speed is increased
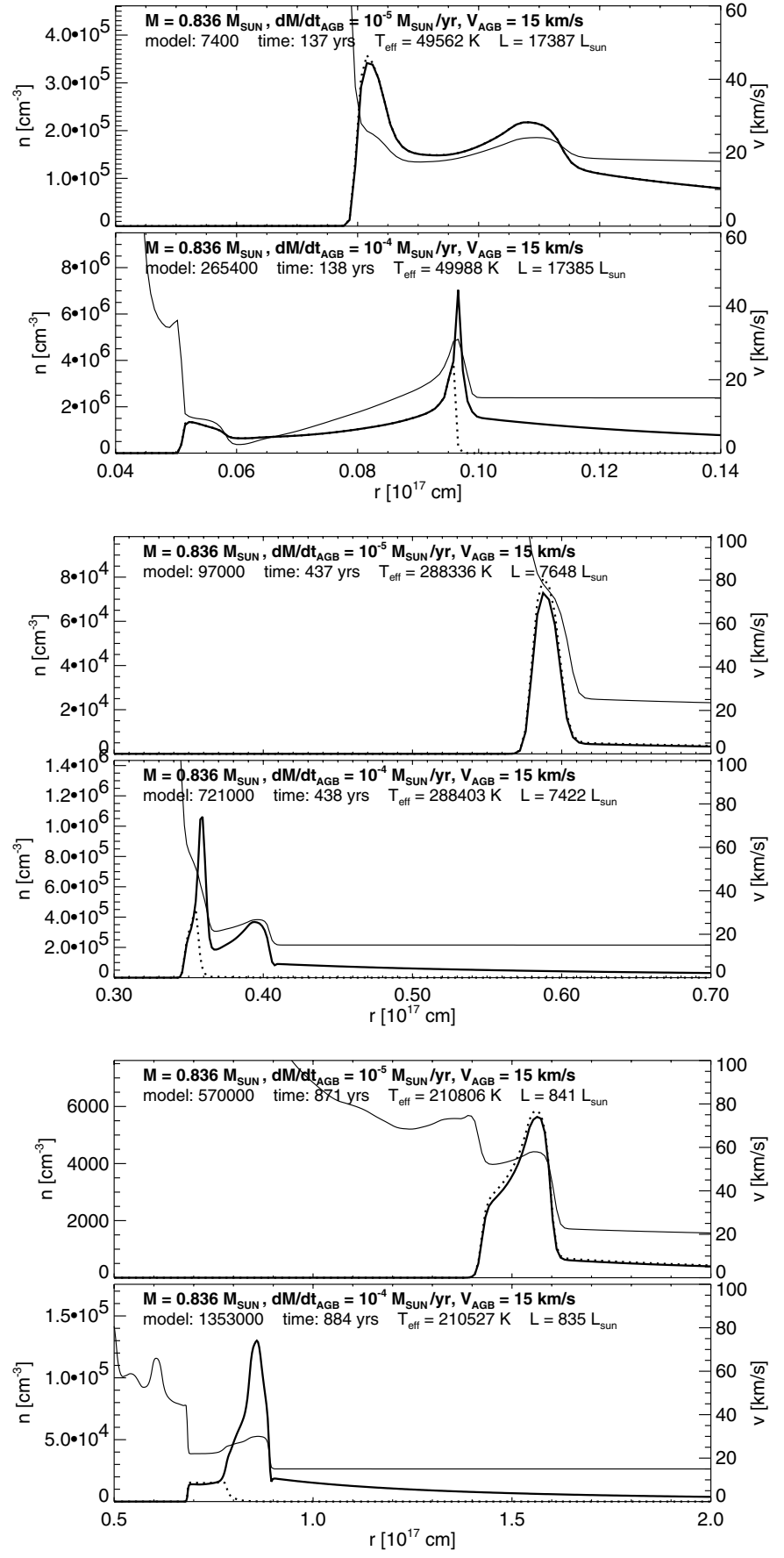

Fig. 13. Models of sequences Nos. 11 and 12 at three evolutionary stages. Line types are the same as before: particle densities (thick), electron densities (dotted), and flow velocities (thin). Note the different range of the radial scales and the flow velocities. Top: compression phase at $T_{\text {eff }} \simeq 50000 \mathrm{~K}$ (PHASE II). Middle: near the turn-around point at maximum compression (PHASE III). Bottom: recombination/reionization at low central-star luminosities (PHASE IV).

by about $6 \mathrm{~km} \mathrm{~s}^{-1}$, and the corresponding density ratio is again about two, irrespective of the different central stars with their very different properties like luminosity and evolutionary speed (for an explanation see the discussion in Sect. 5).

The dense model shows a similar behaviour as previously found in sequence No. 4 (see Sect. 4.1.1): the ionized matter of 
the "shell" recombines already before the turn-around point is reached, and the still (partly) ionized "rim" is surrounded by a now fully neutral shell headed by a strong shock front moving outwards with about $35 \mathrm{~km} \mathrm{~s}^{-1}$ into the ambient, undisturbed AGB wind (Fig. 13, middle panel, top model). This shock wave is the relic of the D-type shock created earlier during the ionization phase (cf. Fig. 13, top panels, top model). The shock compressed neutral AGB matter is being heated up to about $8000 \mathrm{~K}$. The inner part of the "rim" remains ionized, and its pressure accelerates the outer neutral parts to velocities exceeding those in the recombined "shell", i.e. we have a re-ionization front of type D. Such a structure is very similar to that found for an $\mathrm{H}$ II region around a central source with varying UV output, i.e. a star cluster (Beltrametti et al. 1982).

The high-density model shown in Fig. 13 would allow to have shock-excited molecular hydrogen in the immediate vicinity of an optically thick nebular H II region surrounding a very hot stellar object. In this case of more massive and rather quickly evolving central stars we need only a rather short period of high AGB mass loss, i.e. for less than 1000 years. The scenario found here appears to us a rather natural explanation for a long standing problem: the detection of molecular hydrogen (and CO) emission from some planetary nebulae. Judged from our models this molecular emission implies that these PNe have more massive, rapidly evolving central stars.

During the low-luminosity phase, both models continue to develop different structures (Fig. 13, bottom panels). In the dense model, the new D-type shock created after recombination has already overtaken the outer shock and continues to expand supersonically into and to compress and heat the slow and neutral AGB-wind. The H II region has nearly constant density and is surrounded by a dense shell of this swept-up and shockheated neutral AGB material. The less dense model remains fully ionized, but its shell widens and acquires positive density and velocity gradients across the shell during re-ionization $(\Delta R / R \simeq 0.14$ at the particular time shown in the figure). The flow velocities are still quite high, $\simeq 60 \mathrm{~km} \mathrm{~s}^{-1}$ immediately behind the shock.

From the morphological point of view both model sequences develop quickly single shell nebular structures after a short phase of ionization when double-shell structures are prevailing.

\subsection{3. $0.565 M_{\odot}$}

Here we have two sequences with a slowly evolving, lowermass AGB remnant of $0.565 M_{\odot}$ (Nos. 21 and 22 of Table 1). The stellar model needs about 20000 years to reach the turnaround point, and it fades slowly by a factor of ten during the next 20000 years (cf. Fig. 1). The TYPE A envelope models shown in the Fig. 14 are for $\dot{M}_{\mathrm{AGB}}$ of $10^{-5}$ and $3 \times 10^{-5} M_{\odot} / \mathrm{yr}$, resp., with $V_{\mathrm{AGB}}=10 \mathrm{~km} \mathrm{~s}^{-1}$.

Both model nebulae become optically thin well before the central star reaches $30000 \mathrm{~K}$, and they develop the typical double-shell structure like the other models discussed above. Especially the properties of the outer shocks are identical to
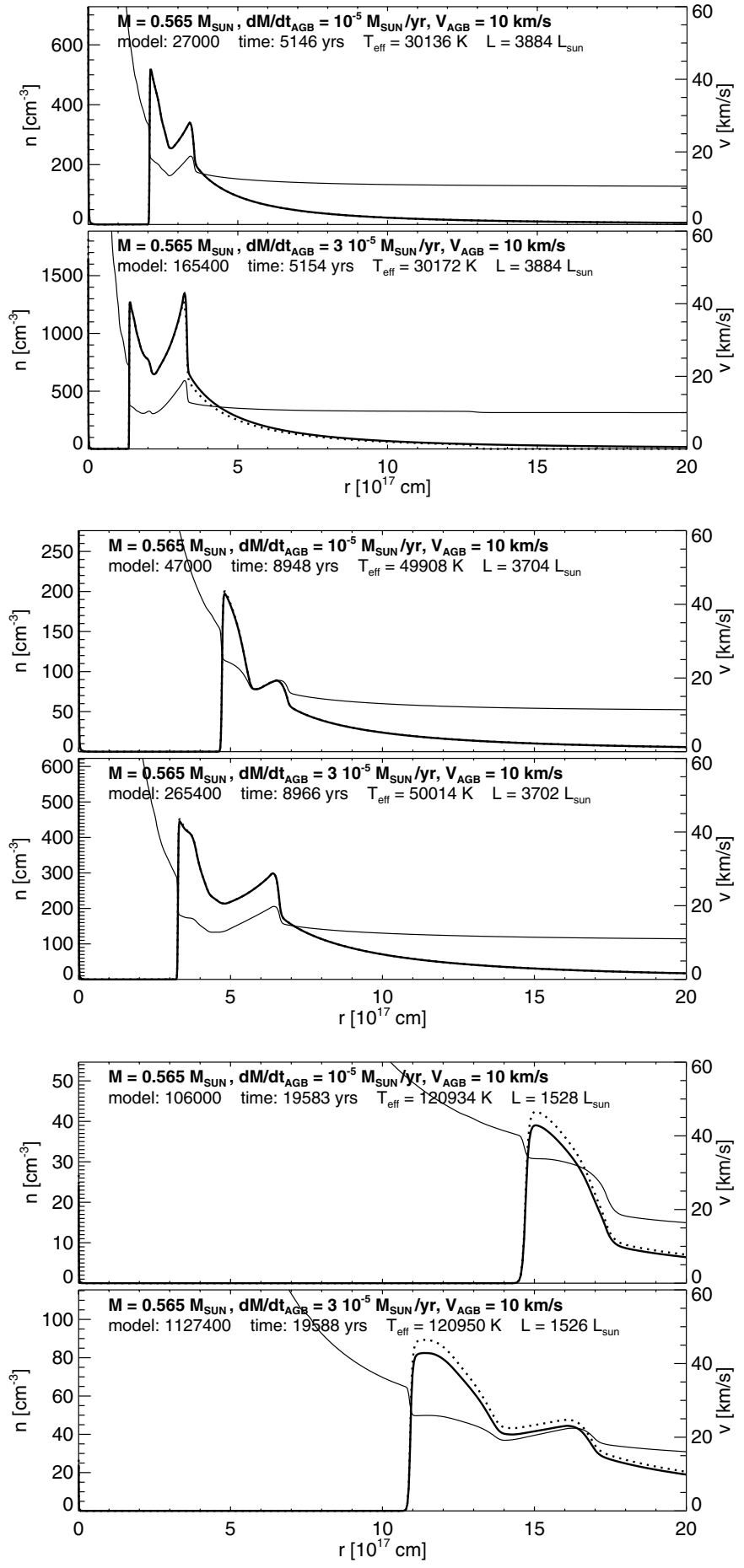

Fig. 14. Models of sequences Nos. 21 and 22 at three evolutionary phases. Top: $T_{\text {eff }} \simeq 30000 \mathrm{~K}$, middle: $T_{\text {eff }} \simeq 50000 \mathrm{~K}$ (PHASE II), and bottom: $T_{\text {eff }} \simeq 121000 \mathrm{~K}$ (turn-around point, PHASE III). Line types have the same meaning as in the previous figures.

those found for the models discussed in Sects. 4.1.1 and 4.1.2: the AGB wind is compressed by a factor of about two, and the post-shock velocities are 5 to $6 \mathrm{~km} \mathrm{~s}^{-1}$ above those of the AGB wind. Driven by the expanding "hot bubble", a dense "rim" of swept-up nebular matter that expands soon faster than the outer shock develops during the later course of evolution, especially in the low-density model (sequence No. 21, middle of Fig. 14, top panel). 


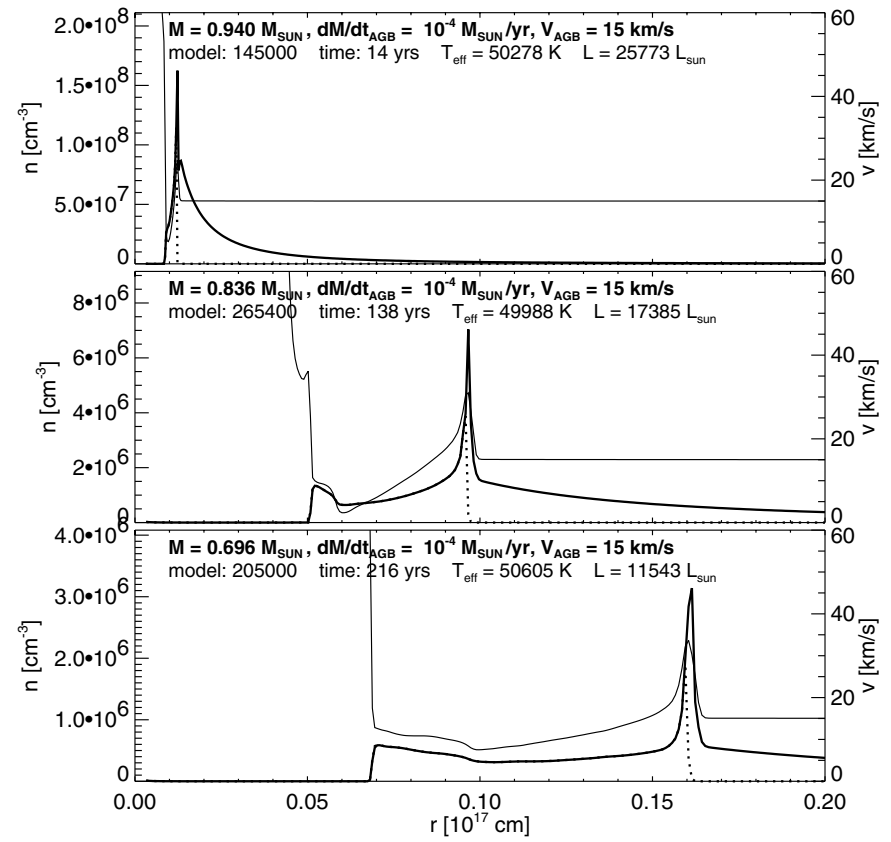

Fig. 15. Nebular structures at $T_{\text {eff }}=50000 \mathrm{~K}$, (PHASE II) for the same AGB starting TYPE A envelope but different central-star masses, from $M=0.940$ (top), $M=0.836$ (middle) to $M=0.696 M_{\odot}$ (bottom), corresponding to sequences Nos. 14, 12, 10 of Table 1 (CASE II).

At the end of our simulation the "shell" has been overtaken by the "rim", obviously the typical destiny if the initial circumstellar envelope densities are rather low and matter can effectively be accelerated by the bubble's pressure (bottom of Fig. 14, top panel). The model consists now of a relatively thin $(\Delta R / R \simeq 0.15)$ shell of compressed and accelerated $\left(V_{\max } \simeq 35 \mathrm{~km} \mathrm{~s}^{-1}\right.$ ) matter. Also the denser of the two models shown is quite compressed $(\Delta R / R \simeq 0.35)$, but the double-shell structure is still existing.

Note that for such rather slowly evolving central stars as shown in Fig. 14, recombination will not occur: the stellar luminosity decreases only slowly, and after about 20000 years of nebular expansion the recombination time scale becomes long because of the low electron densities.

\subsection{CASE II: Different central stars with the same AGB starting model}

The significance of the central star's evolutionary speed can be demonstrated by combining the same initial envelope model with central stars of different mass. For this purpose, we show in the Figs. 15 and 16 selected models of the sequences 14, $12,10,8$, and 5 for PHASE II $\left(T_{\text {eff }}=50000 \mathrm{~K}\right)$ and the TYPE A starting model with $\dot{M}_{\mathrm{AGB}}=10^{-4} M_{\odot} / \mathrm{yr}$ and $V_{\mathrm{AGB}}=$ $15 \mathrm{~km} \mathrm{~s}^{-1}$.

The large differences in the evolutionary speeds are reflected in the post-AGB ages at $50000 \mathrm{~K}$ : they range from 14 years for $0.940 M_{\odot}$ to 2996 years for $0.605 M_{\odot}$, and we encounter huge differences in densities accordingly. Therefore, the very compact circumstellar envelopes around the massive central stars are still completely optically thick (Fig. 15), and they will stay so for the remaining part of their evolution down

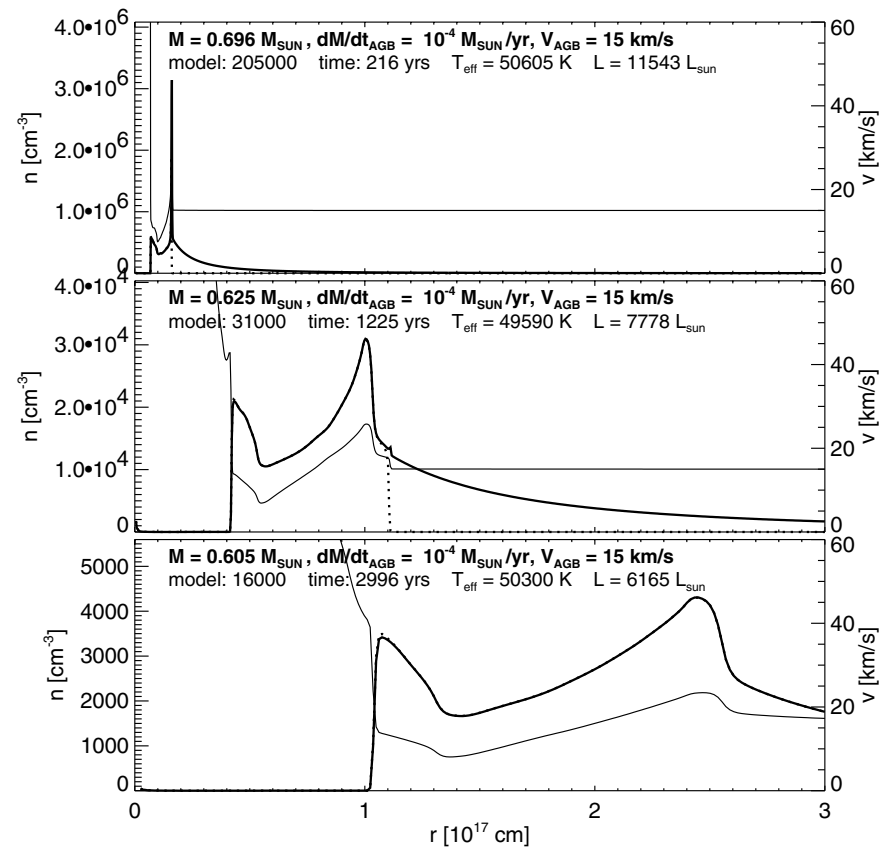

Fig. 16. PhASE II as in Fig. 15, but for lower stellar masses, from $M=0.696$ (top), $M=0.625$ (middle) to $M=0.605 M_{\odot}$ (bottom), corresponding to sequences Nos. $10,8,5$ of Table 1 . Note the different radial coverage. The top frame contains the same model as the bottom frame of Fig. 15 for comparison. Note also that the bottom frame of this figure is the same as in Fig. 10.

to the white-dwarf domain. In the $0.625 M_{\odot}$ model the ionization front has just broken through the outer shock into the neutral AGB wind. Only in the $0.605 M_{\odot}$ case the whole grid is already ionized (cf. Fig. 16, bottom panel). The latter two sequences are, however, subject to recombination when the central stars are rapidly approaching the white-dwarf region.

All models, except the one around $0.940 M_{\odot}$, show already the well-known double-shell structure because the inner edge of the H II region is being compressed by the pressure of the shocked fast central-star wind (i.e. the hot bubble). Likewise, the flow properties are very similar.

The $M=0.625 M_{\odot}$ model constitutes a special case. The PN proper becomes optically thin (cf. Fig. 16, middle panel), but the evolution of the central star is so fast that it competes with the advancing ionization front: the star fades before the grid becomes completely ionized, and a recombination front moves inwards until the inner parts of the "rim" are reached. The ionized part of the halo is accelerated and drives a thin shell of compressed neutral matter (Fig. 17).

\subsection{CASE III: More realistic approaches}

In this section we investigate the question whether more realistic simulations of the AGB mass-loss history may significantly influence the following evolution through the PN stage. For this purpose we computed sequences with different approaches of constructing initial AGB wind envelopes, viz. the TYPES B (seq. Nos. $7 \&$ 9) and C (seq. No. 6), as already explained in Sect. 2.2 and shown in Fig. 2. The models of seq. 6 with the TYPE C initial envelope may be considered to be the most 


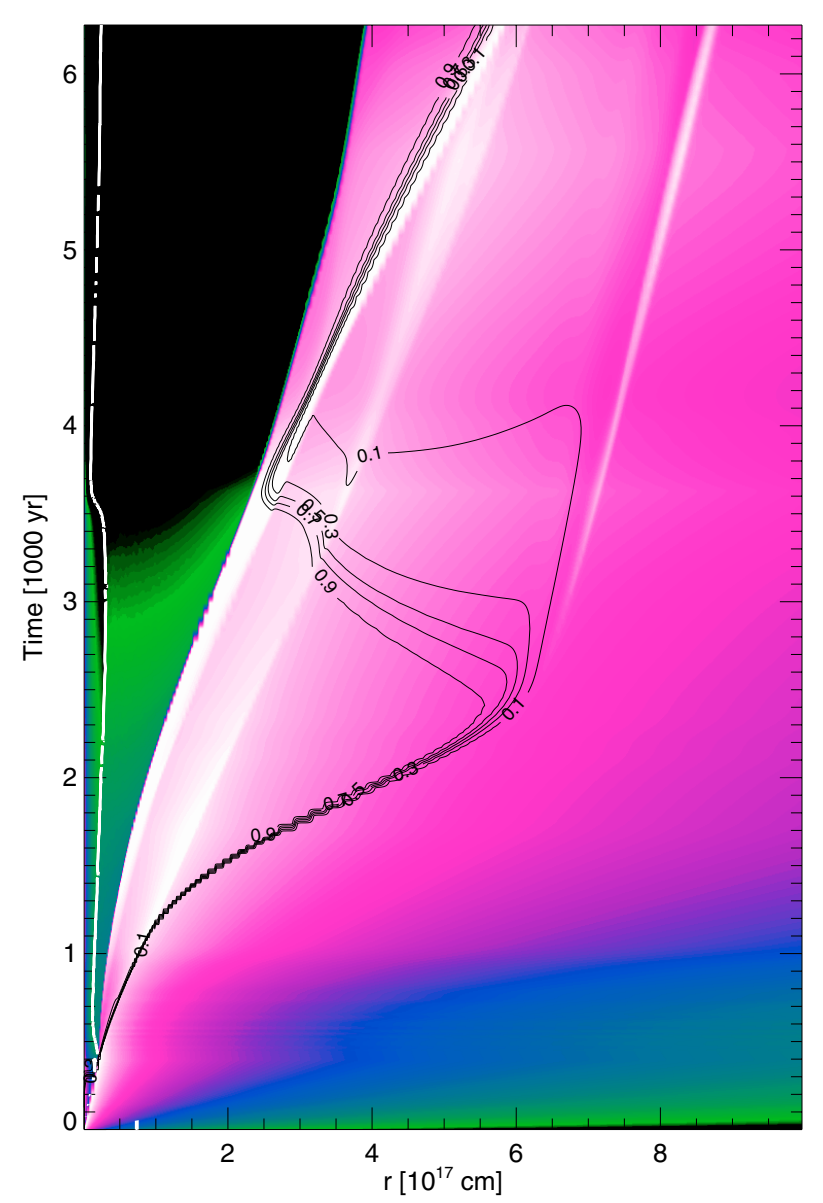

Fig. 17. Position-time diagram of seq. No. $8\left(M=0.625 M_{\odot}\right)$ with the logarithm of the normalized density $\rho(r, t) / \rho_{\max }(t)$ coded as grey scales: highest densities white, lowest densities black. The black contour lines indicate the degree of hydrogen ionization, the white line marks the position of the fast wind shock. The inward hook in the position of this shock at 3600 years is due to the rapid fading of the central star.

realistic approach for describing (spherical) planetary nebulae and has already been used in Perinotto et al. (1998) and Corradi et al. (2000).

\subsubsection{Kinematical approach}

Figures 18 and 19 illustrate the overall evolutionary behaviour of the two TYPE B sequences Nos. $9 \& 7$ listed in Table 1. The models of seq. No. 9 (Fig. 18) with a central star of $M=0.696 M_{\odot}$ and a peak mass-loss rate of $6 \times 10^{-5} M_{\odot} / \mathrm{yr}$ behave like those of seq. No. 12 presented in Sect. 4.1.2 and illustrated in Fig. 13. The evolution across the Hertzsprung-Russell diagram is very short ( $\simeq 1000 \mathrm{yr}$ ), and the following planetarynebula evolution is ruled by a hot but faint white-dwarf central star. The very compact and dense circumstellar envelope prohibits full ionization during the brief high-luminosity phase, and during the following evolution along the hot white-dwarf path the PN remains optically thick for the whole simulation time. The ionized region is preceded by a dense shell of shock-compressed neutral AGB-wind matter, the shock wave being set up during the brief ionizing phase. The shock front is

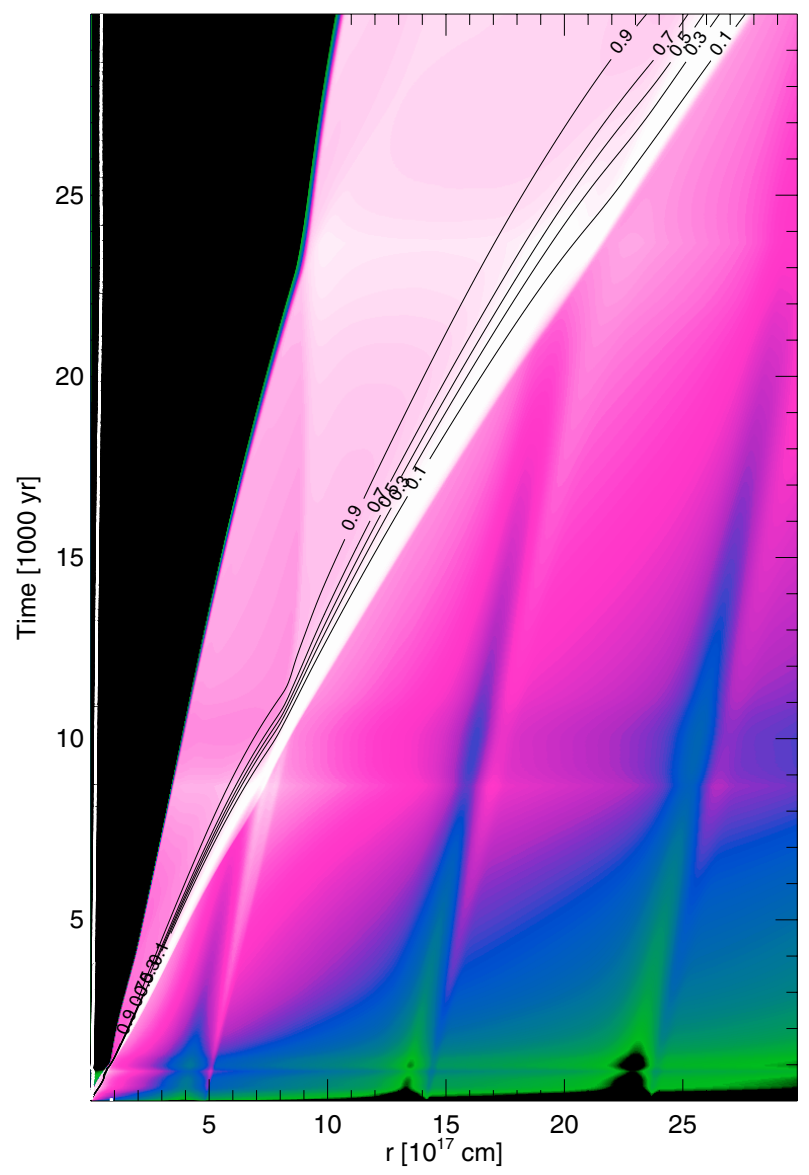

Fig. 18. Same as in Fig. 17, but for seq. No. $9\left(M=0.696 M_{\odot}\right)$. Due to the fast fading of this central-star model, the reverse shock of the fast wind jumps inwards after only 1000 years of post-AGB evolution.

moving with an average (relative) speed of $17 \mathrm{~km} \mathrm{~s}^{-1}$ through the AGB wind, i.e. it expands with $27 \mathrm{~km} \mathrm{~s}^{-1}$ relative to the star. The contact discontinuity expands with only $13 \mathrm{~km} \mathrm{~s}^{-1}$, and is further slowed down later. Thus the size of the H II region increases steadily, reaching a relative shell thickness of about 0.6 at the end of our simulation. As can be seen from the contour lines indicating the degree of ionization, the ionization front remains trapped by the shock for the total time span of our simulation, $\sim 30000$ years.

The difference to the previously discussed cases is that now the AGB-wind density distribution is modulated by thermal pulses, separated in time by $\approx 30000$ yrs. The encounter of the shock front with the signature of the last flash occurs at $t \simeq 8000$ years: the front is slightly accelerated while approaching the density dip, and somewhat decelerated afterwards. The density "spikes" generated by the flash and seen in the initial model (Fig. 2, next to bottom) are broadened by ionization and create an inward running density wave when being swallowed by the PN shock. A similar behaviour is seen when the front reaches the signatures of the next to the last flash. The overall expansion rate is, however, not disturbed by density variations induced by these thermal pulses.

Sequence No. 7, shown in Fig. 19, behaves differently: because of the rather small peak AGB mass-loss rate of only $2 \times 10^{-5} M_{\odot} /$ yr we have a rather low matter density in the 


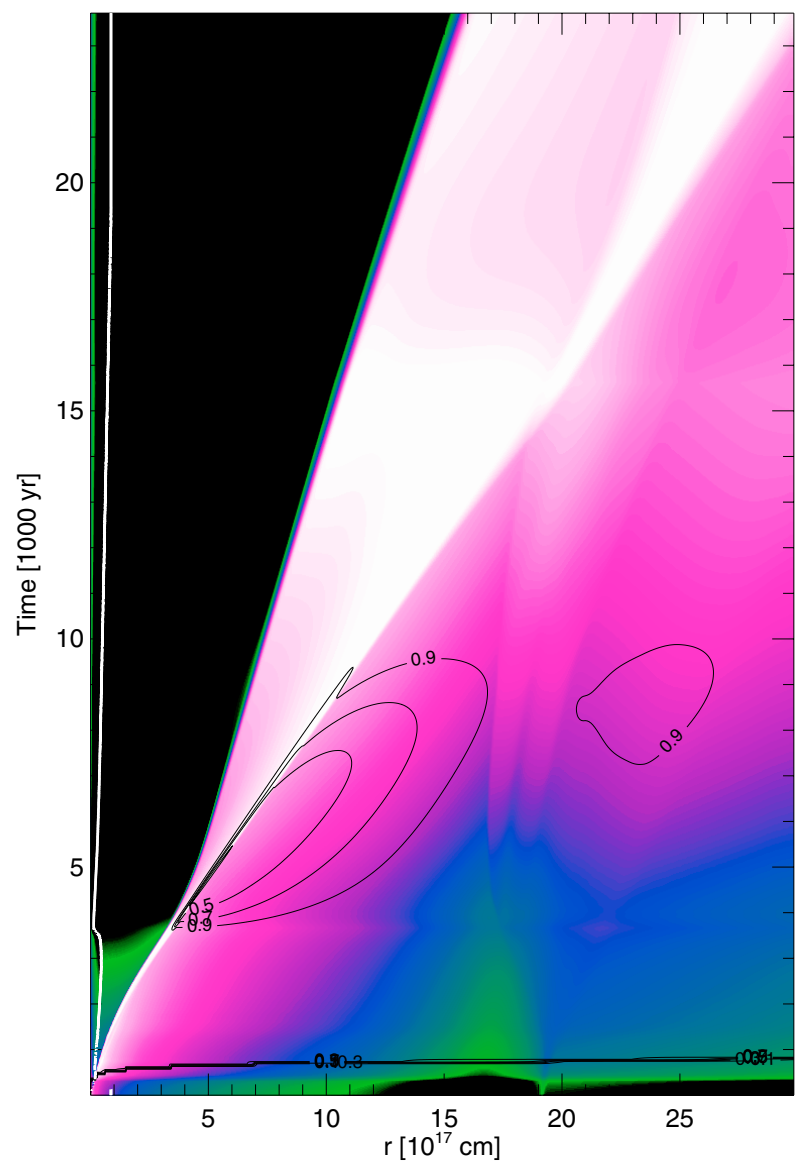

Fig. 19. Same as in Fig. 17, but for seq. No. $7\left(M=0.625 M_{\odot}\right)$. Here the white-dwarf evolution begins at 3000 years after the model has left the tip of the AGB.

vicinity of the star, with only a small density jump at the AGB/post-AGB transition. The ionization front remains of the $\mathrm{R}$ type, and a double-shell configuration does not develop. The whole computational regime is already fully ionized after only 700 years. At the turn-around point after 3500 years we have a dense, compressed ionized shell traveling with a speed of $40 \mathrm{~km} \mathrm{~s}^{-1}$ (relative to the star) through the ionized AGB wind. During the low-luminosity phase the contact discontinuity is slowed down to $20 \mathrm{~km} \mathrm{~s}^{-1}$, and the shell widens because its front continues to travel outwards with high speed (cf. Fig. 4). Recombination is only partial and does not influence the evolution of the PN at all. The signature of the last thermal pulse is at such a large distance from the star that it is reached by the outer shock only in a very advanced phase of evolution.

\subsubsection{Initial model based on detailed hydrodynamical AGB wind envelopes}

The overall evolutionary behaviour of sequence No. 6 with the hydrodynamical initial models is illustrated in Fig. 20. The peak mass-loss rate achieved is $1.4 \times 10^{-4} M_{\odot} / \mathrm{yr}$, and the neutral envelope shows a rich density structure indicative of the mass-loss history during the end of the AGB evolution (cf. Fig. 2): a region of low density caused by a temporary

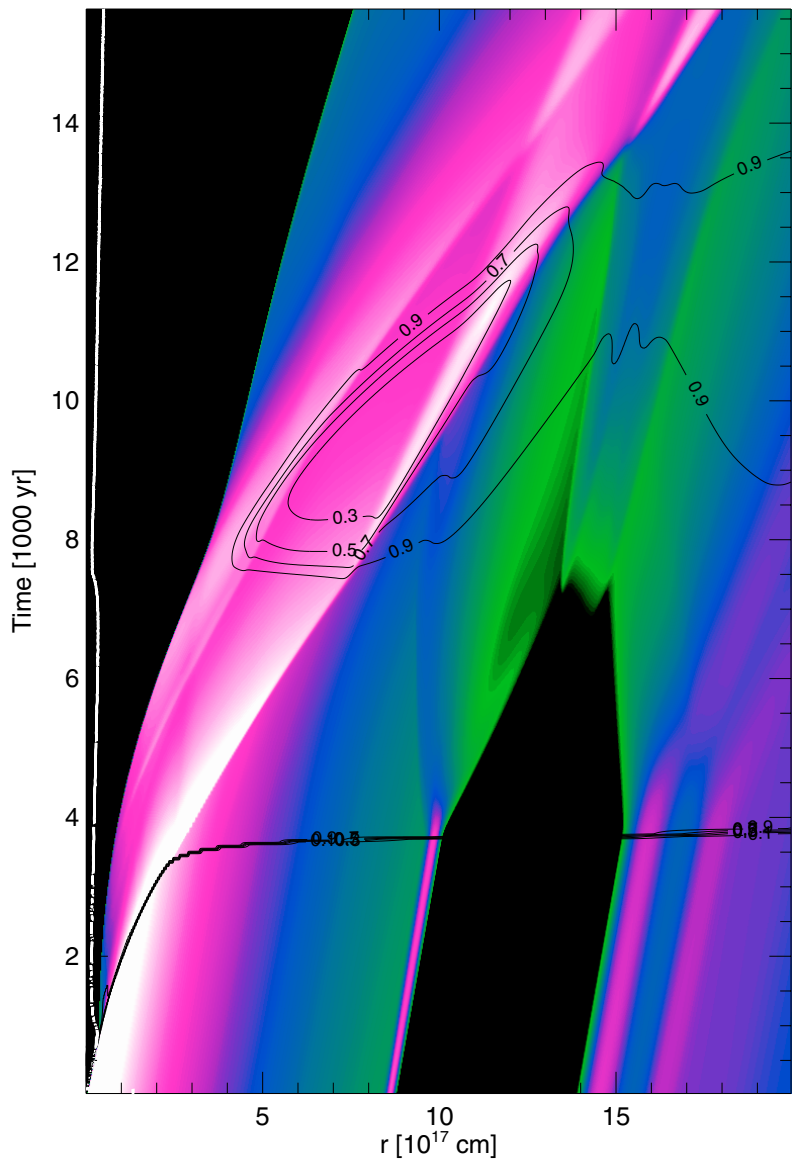

Fig. 20. Same as in Fig. 17, but for seq. 6, the detailed hydrodynamic treatment of the AGB wind envelope for $M=0.605 M_{\odot}$. In this particular case we have plotted the logarithm of density times radius squared, $\rho(r, t) r^{2} / \rho_{\max }(t) r^{2}$, in order to highlight the low-density structures in the halo.

mass-loss interruption following the last thermal pulse on the AGB (see Steffen \& Schönberner 2003 for more details) is bounded by thin shells of higher densities, generated either directly during the thermal pulse (at $\simeq 14 \times 10^{17} \mathrm{~cm}$ ), or by the dynamical pressure of the final strong AGB wind (at $\simeq 9 \times 10^{17} \mathrm{~cm}$ ). Once these shells become heated by photoionization (after $3800 \mathrm{yrs}$ ), they expand because of their increased thermal pressure, setting up a system of inwards and outwards running shock waves modifying thereby the original structure of the AGB wind, now seen as the halo (see again Steffen \& Schönberner 2003).

Figure 21 illustrates the nebular structures at relevant ages in more detail. Firstly we note that this model becomes optically thin late because of the rather large final AGB massloss rates. At $T_{\text {eff }} \simeq 50000 \mathrm{~K}$ shown in Fig. 21 (top panel) it is still optically thick and closely resembles the corresponding model of seq. 4 shown in Fig. 10 which has quite similar values for AGB mass-loss rate and velocity. However, the model shown here becomes optically thin shortly after this stage because the AGB wind density falls off much steeper than $\rho \propto r^{-2}$.

The PN proper develops a rather wide "shell", and its (shock) front reaches a speed, relative to the central star, of $42 \mathrm{~km} \mathrm{~s}^{-1}$ at the turn-around point, the largest expansion rate of 

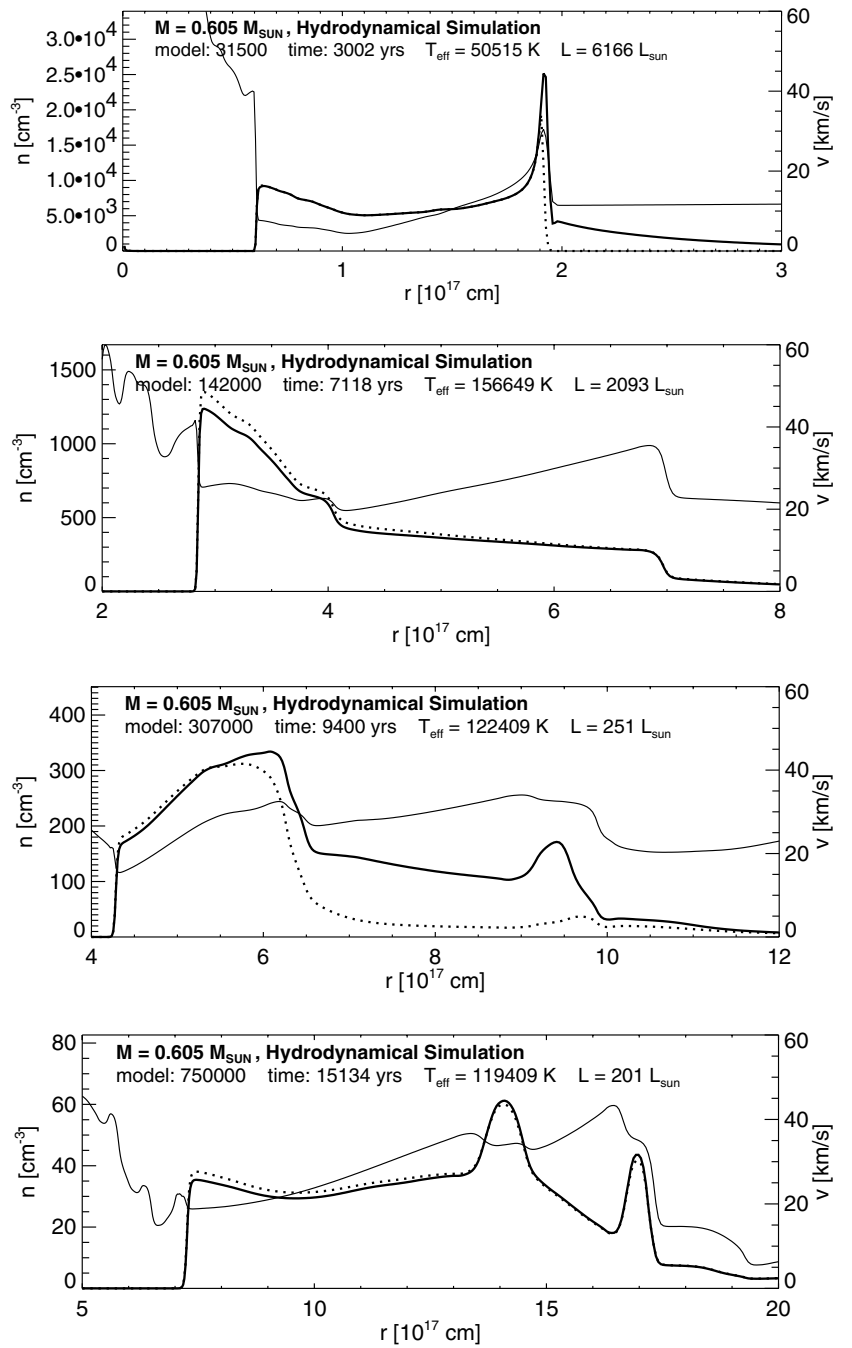

Fig. 21. Density and velocity structure for various snapshots along sequence No. 6 shown in Fig. 20. The stellar parameters are given in the individual panels. The models selected are for a still optical thick case (top), for the turn-around position (next to top), during the recombination phase (next to bottom), and for a very late, reionized stage (bottom).

all of our double-shell models. The flow velocity immediately behind the front is only $35 \mathrm{~km} \mathrm{~s}^{-1}$. This models gains also the largest "shell"-to-"rim" ratio of all our sequences (Fig. 21, next to top). Note also that this model is the only one that develops a negative density gradient within the "shell". These results are caused by the the steep density gradient of the AGB envelope leading to a larger expansion of the outer shock. A more general discussion how expansion rates depend on initial structures will be given below in Sect. 5 .

During the central star's rapid fading (after 7000 yrs) the evolution is similar to the previously discussed cases: the gas of the "shell" recombines to a large extent, and the shock front is slowed down accordingly because of the reduced pressure. The still fast flowing shell material is being compressed into a (geometrically) thin mainly neutral shell (Fig. 21, next to bottom). In the course of the further evolution this neutral shell widens under the action of its thermal pressure, especially when reionization sets in. At the same time the "rim" widens considerably because the expansion of the contact surface is slowed down (see Fig. 20).

This figure also illustrates quite well that in the late evolutionary stages the densities of shells and the expansion speeds of their respective fronts are modified when they cross each other or when they encounter regions of different densities. The bottom panel of Fig. 21 gives the structure of a model in such an advanced stage of evolution: it has a wide "rim" with a nearly constant density, bounded by two thin, compressed shells as a result of such interactions (compare with Fig. 20, at age of 15000 years).

\section{Summary and discussion of the results}

The dynamical system of a planetary nebula as a whole is characterized by three regions that are, counted from outside, the neutral or ionized AGB wind (= halo), the PN proper, bounded from outside by a shock front running into the slow AGB wind and from inside by the contact surface, and the central-star wind which is thermalized through a strong reverse shock. This leads to a gas bubble of high temperature and pressure acting on the the inside of the PN proper.

In all sequences computed, independent of the starting configurations, ionization firstly dominates over the effects of wind interaction and determines the internal structure and observable morphology of a young planetary nebulae. Only later when the central-star wind accelerates and the wind power increases, the inner parts of the $\mathrm{H}$ II region are being compressed into a denser shell, thereby creating the typical double-shell structure: the accelerating contact surface, together with the thermal pressure due to ionization heating creates a structure with large variations of density and velocity gradients across the ionized areas. While the high pressure inside the $\mathrm{H}$ II region accelerates matter outwards and decelerates it inwards, thereby setting up a positive density and velocity gradient for the "shell" flow, the expansion of the bubble compresses and accelerates again the (decelerated) "shell" gas and leads to mainly negative gradients of density and flow velocity within the "rim". The latter is preceded by a weak shock, and gas density and flow velocity have both a local minimum at this "shell"-"rim" interface.

The ionization front starts typically as a D-type front, creating and accelerating a shock wave into the ambient neutral AGB wind because of the huge pressure difference between the ionized and neutral parts of the circumstellar matter. Depending on the AGB-wind density and the flux of ionizing photons, this D-type front may evolve into an R-type one, leaving the density (or pressure) wave behind. The PN is now said to be optically thin. We note that it is this front of the density wave created previously by ionization that defines now the outer edge of the PN. The ionized wind-blown gas surrounding the nebular edge is visible as the AGB halo.

For rapidly evolving central stars with their large circumstellar gas densities the D-type ionization front is not a transient phenomenon, i.e. the change from a D to an R-type front will not occur at all. This property of systems with fast evolving central stars has already been noted by Giuliani (1981) by means of self-similar solutions of the hydrodynamical equations. 
For sufficiently dense models the rapid fading of the central stars towards the white-dwarf domain leads to recombination in the outer PN regions, at least partially. The outer shock front is then slowed down because of the lower pressure of the recombined gas, and the still faster flowing "shell" material is being compressed into a thin shell. Observationally, the main nebular structure consists of a single-shell made of the former windcompressed "rim", but now with a positive density and velocity gradient, as is typical for the first ionization epoch during the beginning of the PN evolution. A new, second "rim", however, does not appear since the bubble's pressure is now too low. In the light of emission lines the recombined parts of the former PN "shell" may appear as a halo-like, limb-brightened structure (cf. Corradi et al. 2000).

For fast evolving central stars the PN may never become optically thin, and in this case recombination occurrs already well before the turn-around point of the evolutionary path is reached. The ionization retreats from the (D-type) shock which, however, continues to expand into the neutral AGB wind. Upon passing this shock, matter is heated to such an extend that emission from $\mathrm{H}_{2}$ is easily possible. The single-shell structure the typical morphology for PNe with more massive central stars that spend most of the nebular lifetime in a low-luminosity state.

We find that the double-shell structure, consisting of the "shell" and the "rim", is a very robust feature, occurring in nearly all of our sequences, irrespective of the initial conditions. This structure may only be destroyed by two events:

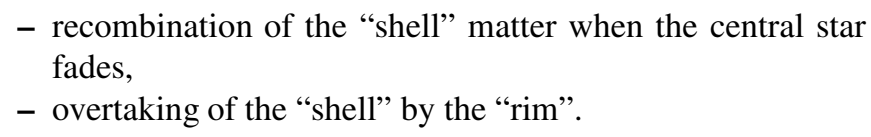

In both cases a single-shell configuration emerges.

The very existence of the double-shell morphology with its two-shock pattern is evidence for the temporal evolution of two different basic processes responsible for the formation of a planetary nebula. The outer shock which is formed first and is defining the "shell", is a manifestation of ionization heating of rather dense AGB-wind matter during the early phase of the nebular formation when the AGB remnant evolves across the H-R diagram. The shock properties as speed and strength depend on the up- and downstream pressure conditions, i.e. mainly on the gas pressure behind and the dynamical (ram) pressure in front, both of which depend on the density slope of the AGB wind envelope. The expansion of a PN thus traces the AGB mass-loss history (cf. Schönberner \& Steffen 1999). On the other hand, the expanding contact surface compresses later the gas left behind the outer shock into a rather thin, dense "rim", preceded by another, normally rather weak, shock whose speed is, again, controlled by the up- and downstream pressure conditions. The expansion of the contact surface is ruled by the (thermal) pressure of the shocked fast wind and thus by the central-star wind history (cf. Schönberner \& Steffen 1999).

From the very beginning of the PN evolution, the two shells develop different and independent flow characteristics. The flow velocity within the "shell" increases linearly with distance from the star with only marginal increase of the maximum speed with time, while the flow velocity within the "rim" is roughly constant or may even decrease with distance from the star. The "rim" develops a significant change of its flow characteristics during the central star's evolution, starting at very low velocities during the ionization phase and ending up with values that may even exceed those of the "shell". The analytical theory of interacting winds as introduced by Kwok et al. (1978) and elaborated in more detail by Volk \& Kwok (1985) is applicable only to the evolution of the "rim"!

We find that the double-shell morphology is always accompanied by a typical "U" or better "V"-shaped velocity field. Interestingly, Gesicki et al. (2003) are claiming to have detected such velocity fields in a number of objects. It must be said, however, that according to Gesicki et al. the corresponding density structures are those of simply compressed shells, completely different from double-shell morphologies which are typical for planetaries of the evolutionary stage in question. Our simulations show that the velocity field of single-shell PN is simple and increases always steadily inwards (cf. Fig. 13, middle panel).

The discussion of the TYPE A configurations in the previous sections revealed the somewhat surprising result that the expansion properties of the models, once the (optically thin) double shell structure is reached, is independent of the initial conditions like AGB mass-loss rate and wind speed: the shock velocities relative to the slow AGB wind and the density jumps across the shock fronts are virtually the same for all the stationary AGB wind models investigated. These properties can be explained by the self-similar properties of "champagne" flows in winds with constant temperatures, as shown by Chevalier (1997) and Shu et al. (2002). These self-similar solutions of the Euler equations for isothermal flows are applicable to our models (and for real planetaries as well) since the shock strengths are rather modest, ensuring nearly constant electron temperatures across the whole ionized region.

As Chevalier and Shu et al. showed, the self-similar solutions for stationary flows, $\rho \propto r^{-2}$, are fully independent of the density but weakly dependent on the absolute flow velocity, and these solutions explain also the radial density and velocity profiles found within the "shells". At each time the self-similar flow pattern behind the shock is characterized as follows: the velocity increases nearly linearly with radius while the density is roughly proportional to distance squared.

Using the parameters relevant for our model sequences, these solutions predict the following expansion properties (Chevalier 1997, Table 1 therein): a shock-front velocity (in the rest frame of the star) of (3-4)× the (isothermal) sound speed, and corresponding post-shock flow velocities between about $(2-3) \times$ the sound speed. The upstream speed (relative to the shock) is $1.4 \times$, the downstream speed $0.75 \times$ that of the sound. The velocity increase across the shock is $(0.5-0.7) \times$ the sound speed, while the gas is compressed by a factor of about 1.7-2.0. Thus the flow acceleration by the shock itself is rather modest. With an isothermal sound speed of about $10 \mathrm{~km} \mathrm{~s}^{-1}$ we get velocity jumps across the shock front of $5-7 \mathrm{~km} \mathrm{~s}^{-1}$, exactly what we have found in our simulations with TYPE A envelopes.

These numbers are strictly valid only if the flow extends to the center, but the change introduced by assuming that the 
flow is driven by an expanding sphere acting at a certain distance from the center is quite small if the expansion is not too fast (Chevalier 1997, Fig. 2 therein). This is the case in our model calculations and explains the close agreement of our hydrodynamical simulations with the numbers given above (cf. Figs. 10, 11 and 14).

Shu et al. (2002) investigated also the more general case of "champagne" flows in environments with $\rho \propto r^{-n}, 3 / 2<n<3$, and found that the shock velocity is an increasing function of the exponent $n$ and diverges for $n \rightarrow 3$. Our hydrodynamical simulation (seq. No. 6, Fig. 2) has a density slope approaching $n \simeq 3$. Although in this case strength and speed of the shock are indeed considerably larger than in the standard TYPE A models, they are not infinite. Most likely the isothermal assumption is no longer valid if speed and shock strength increase, and the flow cannot be described by similarity solutions.

Given a specified exponent $n$, the expansion of a planetary nebula depends then entirely on its thermal properties via the isothermal sound speed. Since the electron temperature, controlled by the heating and cooling processes, varies only slowly during the course of the evolution, the expansion velocities are expected to remain rather modest for the total PN lifetime. More specifically, the electron temperature increases during the optically-thin phase from about $8000 \mathrm{~K}$ towards about $13000 \mathrm{~K}$ later on when the turn-around point is reached (cf. Figs. 6 and 7), corresponding to a less than $30 \%$ increase of the sound speed and hence the expansion velocity.

Furthermore, since the "effective" slope of an AGB wind envelope is ruled by the mass-loss history along the tip of the AGB, the expansion properties of a PN are obviously significant to investigate the very last mass-loss processes on the AGB. Careful measurements of PN expansion velocities may thus shed light upon the temporal behaviour of the AGB mass loss in addition to direct observations of PN haloes.

As has mentioned before, structure and dynamics of the "rim" are different. It is a manifestation of the strong mechanical luminosity of the fast central-star wind and consists of compressed matter from the inner edge of the (ionized) "shell" whose density and speed is determined by the accelerated expansion of the contact surface. The "rim" is led by a shock which is only very weak during the earlier phases (Figs. 6 and 7).

A weak shock propagates with the sound speed, $a$, relative to the ambient flow, $v$, and the shock speed in the rest frame amounts then to $a+v$. For the case that $v \approx a$ as is typical for our models, a rest frame shock speed of $\approx 2 a$ follows. We conclude that the expansion rate of the "rim" may exceed that measured from the flow via the Doppler effect by a factor of about two! In the course of further evolution the shock increases its strength, and the flow speed as well, in such a way that the discrepancy between expansion rate and flow speed decreases.

Figure 22 illustrates the expansion of the "rim" for our hydrodynamical simulation shown in the Figs. 20 and 21. The leading edge of the "rim", defined by the shock, is always considerably faster than the contact discontinuity, i.e. the inner edge of the "rim". The mean "rim" velocity, as measured by means of the Doppler split of emission lines, can be slightly lower than the speed of the contact discontinuity because of

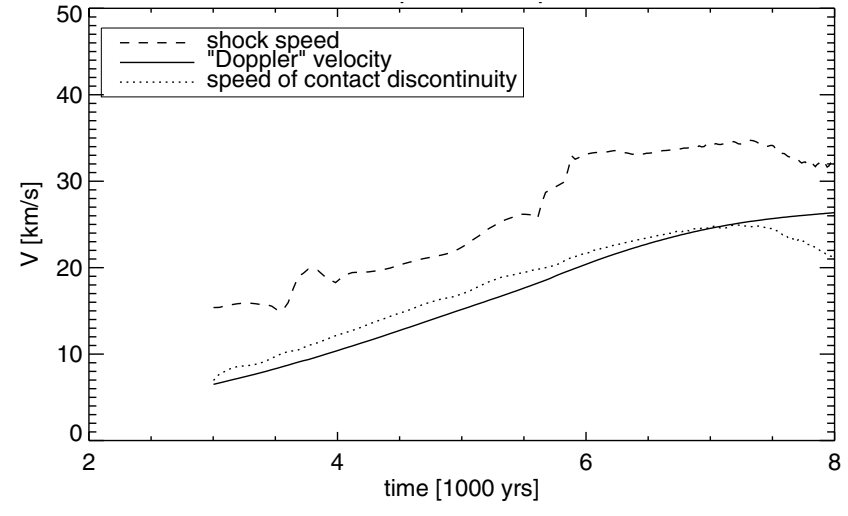

Fig. 22. Expansion properties of the "rim" for seq. No. 6 (hydrodynamical simulation). Shown are the speeds of the contact discontinuity (dotted) and of the leading shock front (dashed) vs. time, together with the "Doppler" velocity (thick). The latter is the mean flow velocity within the "rim", weighted along the central line of sight by density squared and corresponds to the value one would derive from the Doppler split of emission lines. The somewhat erratic velocity changes of the shock front are due to numerical problems in finding the exact shock position. Below 3000 yrs the resp. velocities are not plotted because the whole model is optically thick and the double-shell structure not well developed.

the radial variations of density and velocity (see Fig. 21, top panels). For a substantial span of the model's evolutionary time (for about 1000 years) the shock propagates about twice as fast as one would measure by the Doppler effect, and also in the remaining phases the ratio between shock and "Doppler" speed remains larger than 1.4! Distances to planetary nebulae based on expansion parallaxes are thus systematically too small by a significant amount. A more thorough discussion is beyond the scope of the present paper and will be given in a forthcoming publication.

\section{Comparison with observations}

An important aspect of our hydrodynamical simulation is the detailed comparison with real objects. We thus collected information from the literature about morphology and kinematics of elliptical and round planetary nebulae. The basic criteria for the comparisons are the the relative sizes of "rims" and "shells" together with their surface-brightness distributions, and the respective expansion velocities. In order to ease the comparison of our models with the observations, we computed also observable quantities like surface brightness and emission-line profiles for selected ions.

\subsection{Morphologies}

In a seminal study, Chu et al. (1987) found from a sample of optically thin PNe that the multiple-shell phenomenon is more the rule than the exception. These authors distinguished between limb-brightened, very faint "detached" outer shells (PNe of type I) and the more frequently occurring somewhat brighter but not limb-brightened "attached" shells (PNe of type II). Several objects have both kinds of shells, the limb-brightened 

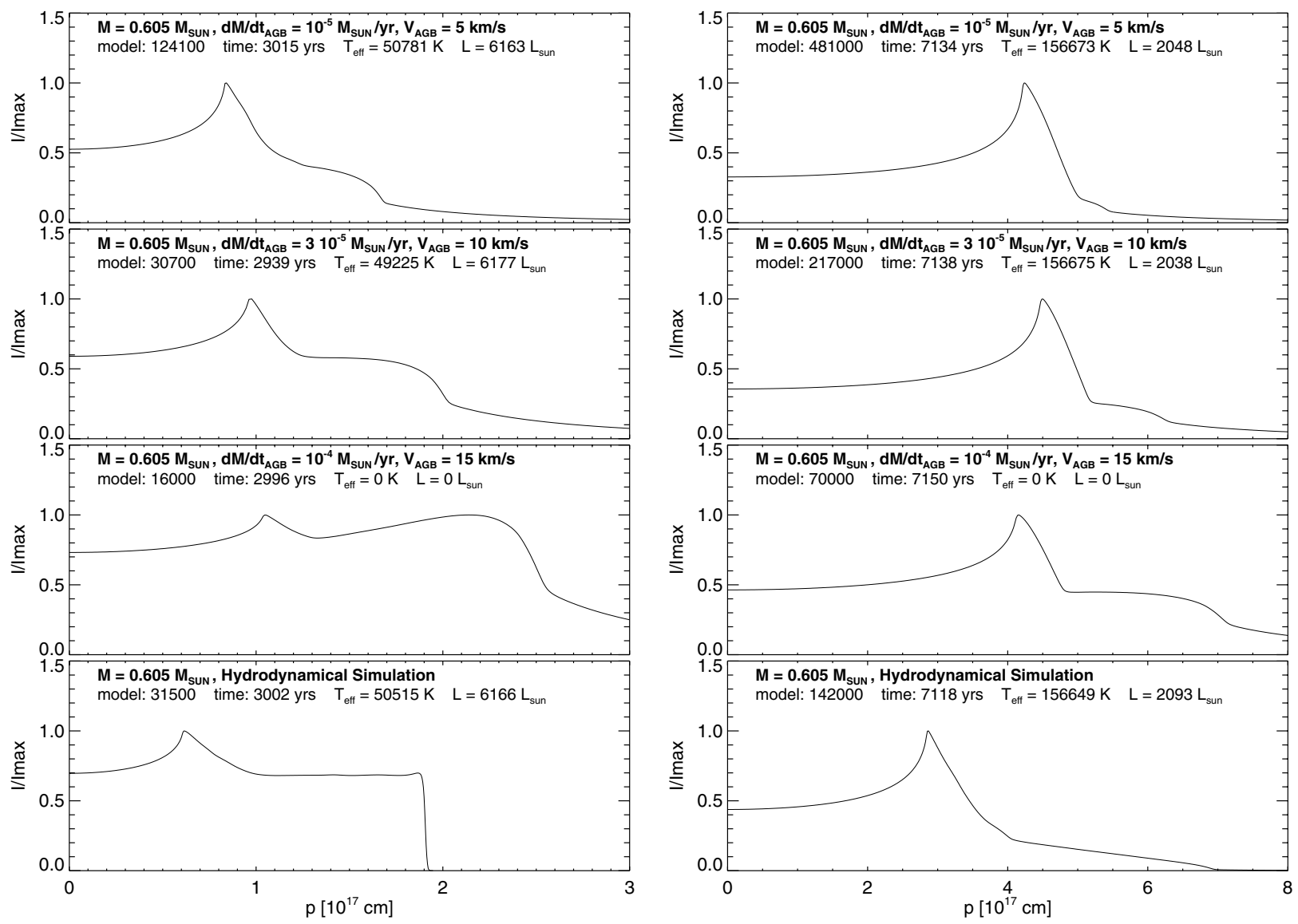

Fig. 23. Surface-brightness in $\mathrm{H} \alpha$ of selected models of the sequences Nos. $2,3,5$, and 6 (from top to bottom, cf. also Table 1), for $T_{\text {eff }} \simeq$ $50000 \mathrm{~K}$ (PHASE II, left) and $T_{\text {eff }} \simeq 156000 \mathrm{~K}$ (PHASE III, right). The corresponding model structures are shown in Figs. 10, 11, and 21, respectively.

of type I being always the outer one. Stanghellini \& Pasquali (1995) enlarged the sample of multiple-shell nebulae by southern objects.

From the evolutionary point of view, a faint "detached" shell is interpreted as the ionized part of the original AGB wind, called a "halo", whereas an "attached" shell is the result of ionization by the hot central star. The properties of the haloes, and their relevance for interpreting the final AGB evolution, have been investigated in detail in a recent study by Corradi et al. (2003). Here, only the properties of the inner main structures are of relevance, i.e. the relative sizes and brightnesses of the (attached) "shells" as compared to the inner bright parts of the PNe, viz. the "rims". The studies of Chu et al. (1987) and Stanghellini \& Pasquali (1995) revealed that the "shells" are greater than the "rims" by factors between roughly 1.5 and 2.3, and much fainter than the latter. Very often the surface brightness of the "shell" decreases linearly with radius (Frank et al. 1990).

The appearance of our models in $\mathrm{H} \alpha$ is illustrated in Fig. 23 for two selected moments. The surface brightnesses reflect the density structure, i.e. we see clearly the double-shell signature together with the emission from the ionized AGB wind, the only exception being the still optically thick model of the hydrodynamical simulation at $T_{\text {eff }}=50000 \mathrm{~K}$ (seq. No. 6).

All the standard TYPE A models do not comply with the observational facts: either the shell is too bright (seq. No. 5), or it is too small (seq. Nos. 2 and 3), and also the halo (AGB wind) emission is too large in most cases. Only the hydrodynamical model shows good agreement with the observations: its "shell" is large and faint, with a surface brightness falling off nearly linearly with distance from the central star, and the halo emission is very faint relative to that of the PN. Also, the size ratio of "shell" and "rim" is well within the observed range.

The initial configuration for the models of seq. No. 6 (Fig. 2, bottom) is the result of detailed hydrodynamical simulations of dust-driven outflows along the AGB where the stellar evolution has been coupled to a mass-loss prescription that depends on the actual stellar parameters (see Steffen et al. 1998). The steep density slope of the initial model is due to the strong increase of the mass-loss rate towards the end of the AGB, as outlined in Sect. 2.2. Such a mass-loss behaviour is supported by recent interpretations of the IR spectral distributions of extreme carbon stars by Volk et al. (2000). These authors found that the density slope is steeper than that for a stationary flow, and concluded that the mass-loss rate must have strongly increased during the last 10000 years towards the end of the AGB evolution. Additionally, surface-brightness images computed with these models agree very well with real objects as far as the general shape and the relative intensities of the shells are concerned (see Schönberner \& Steffen 2003a,b).

Chu et al. (1987) showed that there is also an evolution in the relative shell sizes: the size ratio of "shell" to "rim" 
decreases with size (or age) of the PN (Fig. 24). This figure is an adaptation of Fig. $6 \mathrm{~b}$ in the Chu et al. paper, supplemented by southern objects studied by Stanghellini \& Pasquali (1995). Furthermore, we added spatially resolved LMC objects from the list of Shaw et al. (2001). We retrieved the images from the HST archive and processed them with MIDAS to get the sizes of the individual shells. We preferred the distances provided by Gorny (priv. comm.) which are, on the average, larger than those used by $\mathrm{Chu}$ et al. and Stanghellini \& Pasquali. For the distance to the LMC we used $d=50 \mathrm{kpc}$.

We note further that the data points in Fig. 24 should not be taken too literally. There is first the distance uncertainty (except for the LMC objects), and second the poor definition of individual shell sizes, mainly because the authors do not provide information how they are measured. The 4 LMC PNe displayed in Fig. 24 appear to have the same expansion properties as their Milky Way counterparts. It would be important to prove or to disprove this statement by means of a larger sample of Magellanic Clouds PNe.

Also plotted are relevant model sequences with the parameters indicated in the legend. Despite the uncertainty of the data, we can draw the following conclusions: First, all the simulations agree with the observations in that the shell ratios decrease with size or age of the $\mathrm{PN}^{3}$. Second, judged from the shell ratios and the absolute sizes of the "rims" alone, only a few sequences comply best with the observations, viz. No. $5\left(0.605 / 1 \times 10^{-4} / 15\right)$, No. $6(0.605 /$ hydro $)$, and No. $22\left(0.565 / 3 \times 10^{-5} / 10\right)$. Obviously, peak mass-loss rates significantly below $3 \times 10^{-5} M_{\odot} / \mathrm{yr}$ are not large enough as to make the dynamics of PN work as is observed.

Figure 24 shows also sequence No. $8\left(0.625 / 1 \times 10^{-4} / 15\right)$ as an example of a planetary nebula with a more massive central star. In this case, a double-shell structure develops, and the shell ratio is marginally right, but the absolute sizes remain too small because of the star's rapid evolution across the HR diagram. The situation is worse for planetary nebulae around even more massive central stars: according to our model simulations, they will not evolve at all into double-shell structures.

\subsection{Expansion properties}

The interpretation of observed expansion velocities by means of Doppler splitting of emission lines is somewhat difficult to achieve since at high resolution the lines may display a complex structure, reflecting the corresponding density and velocity structures of the PN in question. It has already been emphasized by Schönberner et al. (1997), based on the structures of hydrodynamical models, that the line splitting at highest intensity reflects the rather uniform expansion of the "rim" material. Most of our models reach, in the course of their evolution, rim velocities between 10 and $30 \mathrm{~km} \mathrm{~s}^{-1}$, quite close to the observed velocity range. Note that most of the expansion velocities listed in existing catalogues refer to line splittings of the main line components, i.e. they give the (weighted) flow velocity within the "rim".

\footnotetext{
${ }^{3}$ At the very beginning of the double-shell phase the shell ratio may increase for a short period.
}

Only a few data exist on the expansion properties of the faint outer "shells". To our knowledge Chu et al. (1984) first pointed out that the nebular emission lines may have faint outer wings or "shoulders", indicating that the "shell" material is moving faster than the main body of the PN. More recent studies of the kinematics of PNe indicate that the flow velocities in the outer regions of double-shell PNe are quite high and can reach $40 \mathrm{~km} \mathrm{~s}^{-1}$ (Gesicki et al. 1996, 1998; Guerrero et al. 1998; Neiner et al. 2000). The corresponding front speeds must then even be larger.

All the TYPE A models fall short of the observed values: the fastest expanding double-shell models are those of seq. No. 5 with the large initial AGB wind speed of $15 \mathrm{~km} \mathrm{~s}^{-1}$, which acquire a post-shock velocity of $27 \mathrm{~km} \mathrm{~s}^{-1}$ at the turn-around point (Fig. 11). This discrepancy between the models and the observations becomes even larger if one considers that the region with the largest (projected) emission measure occurs further inwards where the flow speed is lower (see discussion in Schönberner et al. 1997). Only our CASE C model (hydrodynamical AGB-wind simulation) with its steeper density slope of the AGB wind envelope is faster and complies with $36 \mathrm{~km} \mathrm{~s}^{-1}$ better with the observations (see Fig. 20).

\section{Conclusions}

Our systematic and comprehensive hydrodynamics study of the formation and evolution of planetary nebulae allowed an improved insight into the physical processes ruling the latest stages in the evolution of not too massive stars. Our modeling shows convincingly that even with the simplest initial conditions (constant outflow rate and velocity) the development of a double shell structure is inevitably the consequence of dynamical processes initialized by heating due to photo-ionization in combination with pressure subsequently provided by the shocked fast wind from the central star. Under these conditions it is not possible to derive the mass loss history from the density structure of a PN, as unfortunately frequently seen in the literature, since the PN morphologies have no direct relation to the previous AGB mass-loss histories. Indirectly, however, the density slope of circumstellar AGB-wind envelopes can be estimated by measuring the speed of the flow behind the outer shock front.

The basic points concerning the formation and evolution of planetary nebulae can be summarized as follows:

- The ionization destroys the initial density and velocity distribution by setting up a shock wave traveling outwards.

- The increasing central-star wind power creates a doubleshell structure which may develop into a thin, single-shell configuration if the outer shell is being overtaken.

These more general results are already known from the previous hydrodynamical studies listed in the Introduction. More specific points emerging from the present study are:

- AGB envelopes of lower density evolve, after a short transient double-shell phase, into PNe consisting of (geometrically) thin and fast expanding single shells. 


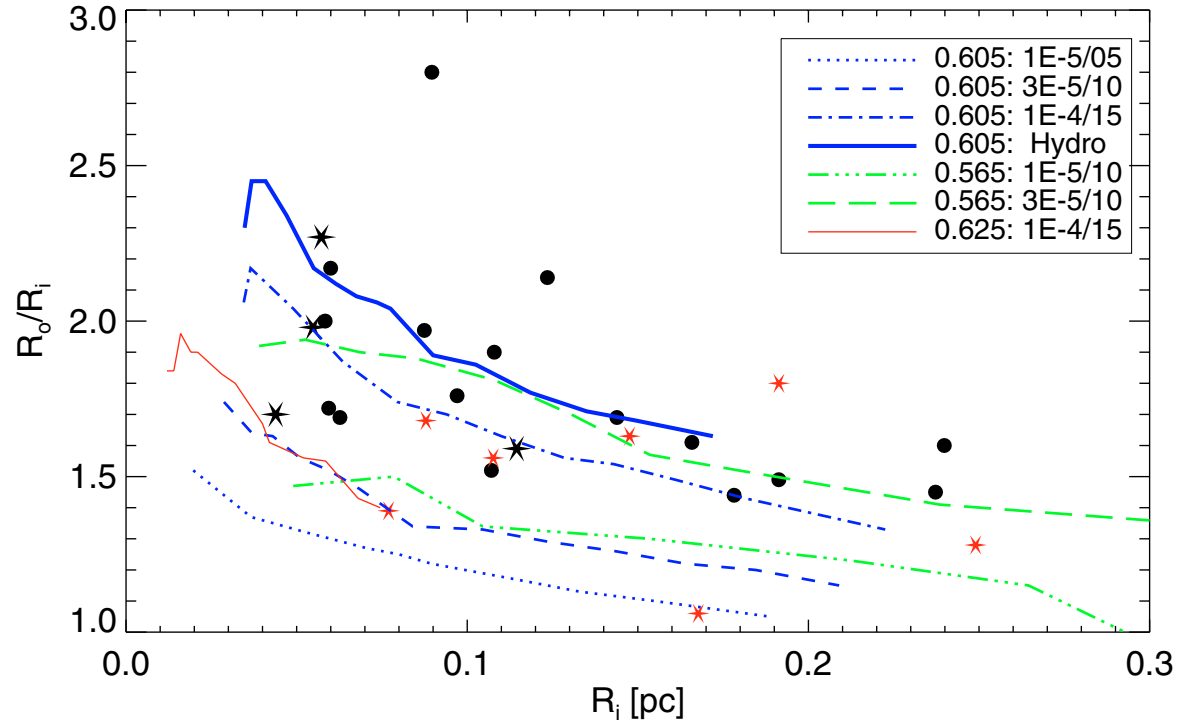

Fig. 24. "Shell" radii, $R_{0}$, over "rim" radii, $R_{\mathrm{i}}$, vs. "rim" radii for double-shell $\mathrm{PNe}$ as adapted from Chu et al. (1987) (filled circles). The data set is supplemented by southern objects collected by Stanghellini \& Pasquali (1995) (small asterisks), and by 4 LMC objects drawn from the list of Shaw et al. (2001) (large asterisks). The theoretical predictions as taken from our different hydrodynamical simulations are shown for comparison. The parameters of the sequences are given in the legend: central-star mass, mass-loss rate (in $M_{\odot} / \mathrm{yr}$ ), and AGB-wind velocity (in $\mathrm{km} \mathrm{s}^{-1}$ ).
- Systems with very fast evolving, massive central stars will never become optically thin during the high-luminosity phase of their evolution, except in the unlikely case of lowdensity AGB envelopes.

- From the sizes of the shells and their evolution with time we found that systems consisting of central stars with masses between 0.60 and $0.57 M_{\odot}$ and AGB-wind envelopes corresponding to mass-loss rates between about $3 \times 10^{-5}$ and $10^{-4} M_{\odot} / \mathrm{yr}$ fit the observations best.

- Large "superwind"-like rates must continue until the remnant star detaches quickly from the AGB, which happens at effective temperatures above $5000 \mathrm{~K}$. Otherwise doubleshell structures will not develop at all since matter densities around the star will become too small (cf. case of seq. No. 7).

- All the models with stationary AGB winds, i.e. with $\rho \propto$ $r^{-2}$, do not acquire the high expansion velocities observed, and also their surface-brightness distributions are at variance with the observations. A larger density gradient is necessary to provide an additional acceleration of the outer shock and make the "shell" more extended and fainter. Thus morphology and expansion properties of double-shell PNe indicate that the mass loss must always increase considerably towards the end of the AGB evolution. This result is in line with that of Mellema (1994) who also found that a density drop in the AGB wind steeper than $r^{-2}$ is necessary in order to get brightness distributions within the "shell" more similar to the observed ones.

Acknowledgements. We gratefully acknowledge an extensive use of CPU time with the IBM SP3 parallel machine of the CINECA computing center (Casalecchio di Reno (BO), Italy) under the agreement between CINECA and CNAA. We thank also C. Jänicke for his help in retrieving and processing the HST images.

\section{References}

Beltrametti, M., Tenorio-Tagle, G., \& Yorke, H. W. 1982, A\&A, 112, 1 Blöcker, T. 1995a, A\&A, 297, 727

Blöcker, T. 1995b, A\&A, 299, 755
Blöcker, T. 2001, Ap\&SS, 275,

Breitschwerdt, D., \& Kahn, F. D. 1990, MNRAS, 244, 521

Bobrowsky, M., \& Zipoy, D. M. 1989, ApJ, 347, 307

Bujarrabal, V., Castro-Carrizo, A., Alcolea, J., \& Sanches Contreras, C. 2001, A\&A, 377,868

Calonaci, C. 2000, Thesis (unpublished)

Calonaci, C., Malfetti., P., \& Perinotto, M. 2001, Science and Supercomputing at Cineca, Report-2001, 264

Cerruti-Sola, M., \& Perinotto, M. 1985, ApJ, 291, 237

Chevalier, R. A. 1997, ApJ, 488, 263

Chu, Y.-H., Kwitter, K. B., Kaler, J. B., \& Jacoby, G. H. 1984, PASP, 96, 598

Chu, Y.-H., Jacoby, G. H., \& Arendt, R. 1987, ApJS, 64, 529

Corradi, R. L. M., Schönberner, D., Steffen, M., \& Perinotto, M. 2000, A\&A, 354, 1071

Corradi, R. L. M., Schönberner, D., Steffen, M., \& Perinotto, M. 2003, MNRAS, 340, 417

Ferch, R. L., \& Salpeter, E. E. 1975, ApJ, 202, 195

Frank, A. 1994, AJ, 107, 261

Frank, A., Balick, B., \& Riley, J. 1990, AJ, 100, 1903

Frank, A., \& Mellema, G. 1994a, A\&A, 289, 937

Frank, A., \& Mellema, G. 1994b, ApJ, 430, 800

Fuji, T., Nakada, Y., \& Parthasarathy, M. 2001, in Post-AGB Objects as a Phase of Stellar Evolution, ed. R. Szczerba, S. K. \& Górny, Astrophys. Space Science Lib., 265, 45

García-Segura, G., Langer, N., Rozyczka, M., \& Franco, J. 1999, ApJ, 517,767

Gesicki, K., Acker, A., \& Szczerba, R. 1996, A\&A, 309, 907

Gesicki, K., Acker, A., \& Zijlstra 2003, A\&A, 400, 957

Gesicki, K., Zijlstra, A., Acker, A., \& Szczerba, R. 1998, A\&A, 329, 265

Guerrero, M. A., Villaver, E., \& Manchado, A. 1998, ApJ, 507, 889

Giuliani, J. L. Jr. 1981, ApJ, 245, 903

Heap, S., Boggess, A., Holm, A., et al. 1978, Nature, 275, 385

Herwig, F. 2001, Ap\&SS, 275, 15

Hoogzaad, S. N., Molster, F. J., Dominik, C., et al. 2003, A\&A, 389, 547

Hrivnak, B. J., Kwok, S., \& Volk, K. M. 1989, ApJ, 346, 265

Icke, V. 1988, A\&A, 202, 177

Icke, V. 1991, A\&A, 251, 369

Icke, V., Balick, B., \& Frank, A. 1992, A\&A, 253, 224

Kahn, F. D., \& Breitschwerdt, D. 1990, MNRAS, 242, 505 
Kudritzki, R. P. 1998, in Stellar Astrophysics for The Local Group, ed. A. Aparicio, A. Herrero, \& F. Sanchez (Cambridge Univ. Press), 149

Kudritzki, R. P., Pauldrach, A., Puls, J., \& Abbott, D. C. 1989, A\&A, 219, 205

Kudritzki, R. P., Mendez, R. H., Puls, J., \& McCarthy, J. K. 1997, in Planetary Nebulae, ed. H. J. Habing, \& H. J. G. L. M. Lamers, (Drodrecht: Kluwer), IAU Symp., 180, 64

Kudritzki, R. P., \& Puls, J. 1999, ARA\&A, 38, 613

Kwok, S., Purton, C. R., \& FitzGerald, P. M. 1978, ApJ, 219, L125

Marigo, P., Girardi, L., Groenwegen, M. A. T., \& Weiss, A. 2002, A\&A, 378, 958

Marten, H. 1993, Ph.D. Thesis, Univ. Kiel

Marten, H. 1995, in Asymmetrical Planetary Nebulae, ed. A. Harpaz, $\&$ N. Soker, Annals of the Israel Physical Society, 11, 273

Marten, H., \& Schönberner, D. 1991, A\&A, 248, 590

Marten, H., \& Szczerba, R. 1997, A\&A, 325, 1132

Mathews, W. G. 1966, ApJ, 143, 173

Mellema, G. 1994, A\&A, 290, 915

Mellema, G. 1995, MNRAS, 277, 173

Mellema, G. 1997, A\&A, 321, L 29

Mellema, G., \& Frank, A. 1995, MNRAS, 273, 401

Mellema, G., Eulderink, F., \& Icke, V. 1991, A\&A, 252, 718

Neiner, C., Acker, A., Gesicki, K., \& Szczerba, R. 2000, A\&A, 358, 321

Okorokov, V. A., Shustov, B. M., Tutukov, A. V., \& Yorke, H. W. 1985, A\&A, 142, 441

Patriarchi, P., \& Perinotto, M. 1991, A\&AS, 91, 325

Pauldrach, A., Puls, J., Kudritzki, R. P., Mendez, R. M., \& Heap, S. H. 1988, A\&A, 207, 123

Perinotto, M. 1993, in Planetary Nebulae, ed. R. Weinberger, \& A. Acker (Dordrecht: Kluwer), IAU Symp., 155, 57

Perinotto, M., Kifonidis, K., Schönberner, D., \& Marten, H. 1998, A\&A, 332, 1044

Reimers, D. 1975, in Problems in Stellar Atmospheres and Envelopes, ed. B. Baschek, W. H. Kegel, \& G. Traving (Berlin: Springer), 229

Schmidt-Voigt, M., \& Köppen, J. 1987a, A\&A, 174, 211

Schmidt-Voigt, M., \& Köppen, J. 1987b, A\&A, 174, 223

Schönberner, D. 1981, A\&A, 103, 119
Schönberner, D. 1983, ApJ, 273, 708

Schönberner, D. 1986, A\&A, 169, 189

Schönberner, D., \& Blöcker, T. 1993, in Luminous High-Latitude Stars, ed. D. Sasselov, ASP Conf. Ser., 45, 357

Schönberner, D., Steffen, M., Stahlberg, J., Kifonidis, K., \& Blöcker, T. 1997, in Advances of Stellar Evolution, ed. R. T. Rood, \& A. Renzini (Cambridge Univ. Press), 146

Schönberner, D., \& Steffen, M. 1999, in Optical and Infrared Spectroscopy of Circumstellar Matter, ed. E. W. Guenther, \& S. Klose, ASP Conf. Ser., 188, 281

Schönberner, D., \& Steffen, M. 2001, in Post-AGB Objects as a Phase of Stellar Evolution, ed. R. Szczerba, \& S. K. Górny (Kluwer Acad. Publ.), 85

Schönberner, D., \& Steffen, M. 2003a, in IAU Symp. 209, Planetary nebulae: Their Evolution and Role in the Universe, ed. S. Kwok, M. Dopita, \& R. Sutherland, ASP Conf. Ser., in press

Schönberner, D., \& Steffen, M. 2003b, in White Dwarfs, ed. D. de Martino, R. Silvotti, J.-E. Solheim, \& R. Kalytis (Kluwer Acad. Publ.), 19

Shaw, R. A., Stanghellini, L., Mutchler, M., Balick, B., \& Blades, J. C. 2001, ApJ, 548, 727

Shu, F. H., Lizano, S., Galli, D., Cantó, \& Laughlin, G. 2002, ApJ, 580, 969

Sofia, S., \& Hunter, Jr. J. H. 1968, ApJ, 152, 405

Stanghellini, L., \& Pasquali, A. 1995, ApJ, 452, 286

Steffen, M., \& Schönberner, D. 2003, in IAU Symp., No. 209, Planetary nebulae: Their Evolution and Role in the Universe, ASP Conf. Ser., in press

Steffen, M., Szczerba, R., \& Schönberner, D. 1998, A\&A, 337, 149

Tylenda, R. 1986, A\&A, 156, 217

Vassiliadis, E., \& Wood, P. R. 1993, ApJ, 413, 641

Vassiliadis, E., \& Wood, P. R. 1994, ApJS, 92, 125

Villaver, E., García-Segura, G., \& Manchado, A. 2002a, ApJ, 571, 880

Villaver, E., Manchado, A., \& García-Segura, G. 2002b, ApJ, 581, 1204

Volk, K., \& Kwok, S. 1985, A\&A, 153, 79

Volk, K., Xiong, G.-Z., \& Kwok, S. 2000, ApJ, 530, 408

Wachter, A., Schröder, K. P., Winters, J. M., Arndt, T. U., \& Sedlmayr, E. 2002, A\&A, 384, 452 